\title{
FLUCTUATION OF SOLUTIONS TO LINEAR ELLIPTIC EQUATIONS WITH NOISY DIFFUSION COEFFICIENTS
}

\author{
ANTOINE GLORIA
}

\begin{abstract}
We consider a linear elliptic equation in divergence form on a bounded domain (or on $\mathbb{R}^{d}$ ) in dimension $d \geq 2$, whose coefficients are perturbed by a stationary noise of correlation length $\varepsilon>0$. We give estimates on the fluctuation of the solution in function of the correlation length $\varepsilon$ of the noise, both in terms of strong $L^{2}$ and weak $L^{1}$ norms. This result can be seen as a quantification of the propagation of uncertainties in linear elliptic partial differential equations.
\end{abstract}

Keywords: quantification of uncertainty, fluctuation, stochastic perturbation, variance estimate, stochastic homogenization.

2000 Mathematics Subject Classification: 35B27, 35J15, 39A70, 60H25, 60F99

\section{Motivation And informal Statement of the ReSults}

Let $A$ be a uniformly elliptic conductivity function on a smooth bounded domain $D$ of $\mathbb{R}^{d}$, and let $f \in L^{2}(D)$. We consider the elliptic equation in $D$

$$
\left\{\begin{array}{rll}
-\nabla \cdot A \nabla u= & \text { in } D \\
u=0 & \text { on } \partial D
\end{array}\right.
$$

and its unique weak solution $u$ in $H_{0}^{1}(D)$. We now perturb the conductivity $A$ by some stochastic stationary noise $B_{\varepsilon}$ with correlation length $\varepsilon>0$ (namely we assume that $B_{\varepsilon}(x)$ and $B_{\varepsilon}(y)$ are independent for all $\left.|x-y|>\varepsilon\right)$, and such that $A+B_{\varepsilon} \in L^{\infty}\left(D, \mathcal{A}_{\alpha \beta}\right)$ almost surely (i. e. $A+B_{\varepsilon}$ is uniformly elliptic with constant $\alpha>0$, and uniformly bounded with constant $\beta \geq \alpha$ ). The weak solution $u_{\varepsilon} \in H_{0}^{1}(D)$ to

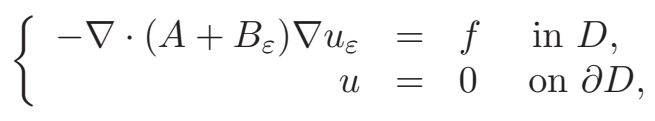

is not deterministic any longer: It fluctuates according to the noise $B_{\varepsilon}$. We wish to quantify the fluctuation of $u_{\varepsilon}$ around its mean value $\left\langle u_{\varepsilon}\right\rangle$ (where $\langle\cdot\rangle$ denotes the expectation with respect to the noise) in function of the correlation length $\varepsilon$ of $B_{\varepsilon}$. To this aim, we consider two different norms for the fluctuation: The (strong) $L^{2}(D)$ norm of the variance, that is

$$
\begin{aligned}
\mathcal{F}_{s}(\varepsilon) & =\int_{D} \operatorname{var}\left[u_{\varepsilon}(x)\right] d x \\
& =\left\langle\int_{D}\left(u_{\varepsilon}(x)-\left\langle u_{\varepsilon}(x)\right\rangle\right)^{2} d x\right\rangle ;
\end{aligned}
$$

Date: July 20, 2012. 
and the variance of a weak $L^{2}(D)$ norm, that is

$$
\begin{aligned}
\mathcal{F}_{w}(\varepsilon, g) & :=\operatorname{var}\left[\int_{D} g(x) u_{\varepsilon}(x) d x\right] \\
& =\left\langle\left(\int_{D} g(x)\left(u_{\varepsilon}(x)-\left\langle u_{\varepsilon}(x)\right\rangle\right) d x\right)^{2}\right\rangle,
\end{aligned}
$$

for any fixed $g \in L^{2}(D)$.

What scaling can we expect for $\mathcal{F}_{s}(\varepsilon)$ and $\mathcal{F}_{w}(\varepsilon, g)$ ? We begin with the strong norm, and interprete problem (1.2) in terms of stochastic homogenization (see for instance [15]). To this aim, we consider $A \equiv \mathrm{Id}$. Let us then denote by $\Phi$ the corrector vector associated with $\left(\mathbf{e}_{1}, \ldots, \mathbf{e}_{d}\right)$ (where $\left\{\mathbf{e}_{1}, \ldots, \mathbf{e}_{d}\right\}$ is the canonical basis of $\mathbb{R}^{d}$ ). From a formal two scale expansion, one has

$$
u_{\varepsilon}(x, \omega)=u_{0}(x)+\varepsilon \Phi\left(\frac{x}{\varepsilon}, \omega\right) \cdot \nabla u_{0}(x)+\ldots,
$$

where $u_{0}$ is the (deterministic) solution to the homogenized equation in $D$

$$
-\nabla \cdot A_{\text {hom }} \nabla u_{0}=f .
$$

In the periodic case (that is, when $B_{\varepsilon}$ is assumed to be $\varepsilon$-periodic instead of stationary), it is known that the error between $u_{\varepsilon}$ and the first two terms of the expansion (1.3) is of order $\varepsilon^{3 / 2}$ in $L^{2}(D)$. One cannot hope better in our stochastic setting, and we momentarily assume this also holds here. In dimension $d>2$, Otto and the author have proved that $\Phi$ can be chosen stationary, $\langle\Phi\rangle=0$, and $\left\langle|\Phi|^{2}\right\rangle<\infty$ (see [6, Corollary 2]). Hence, by the triangle inequality,

$$
\begin{aligned}
\left\langle\int_{D}\left(u_{\varepsilon}(x)-\left\langle u_{\varepsilon}(x)\right\rangle\right)^{2} d x\right\rangle^{1 / 2} & \\
\leq & \left\langle\int_{D}\left(u_{\varepsilon}(x)-u_{0}(x)\right)^{2} d x\right\rangle^{1 / 2}+\left\langle\int_{D}\left(\left\langle u_{\varepsilon}(x)\right\rangle-u_{0}(x)\right)^{2} d x\right\rangle^{1 / 2} \\
\stackrel{(1.3)}{=} & \left\langle\varepsilon^{2} \int_{D}\left(\Phi\left(\frac{x}{\varepsilon}, \omega\right) \cdot \nabla u_{0}(x)\right)^{2} d x+O\left(\varepsilon^{3}\right)\right\rangle^{1 / 2} \\
& +\left(\int_{D}\left\langle u_{\varepsilon}(x)-u_{0}(x)-\varepsilon \Phi\left(\frac{x}{\varepsilon}, \omega\right) \nabla u_{0}(x)\right\rangle^{2} d x\right)^{1 / 2} \\
\leq & \varepsilon\left\langle|\Phi|^{2}\right\rangle^{1 / 2}\left\|\nabla u_{0}\right\|_{L^{2}(D)}+O\left(\varepsilon^{3 / 2}\right) .
\end{aligned}
$$

For $d>2$, we may thus expect $\mathcal{F}_{s}(\varepsilon) \sim \varepsilon^{2}$. This is in agreement with the intuition that $\left(\mathcal{F}_{s}(\varepsilon)\right)^{1 / 2}$ is a measure of a "Poincaré constant" in probability on a domain of lengthscale $\varepsilon$. Note that for $d=1$, explicit calculations show that $\mathcal{F}_{s}(\varepsilon) \sim \varepsilon$. In particular, the corrector cannot be stationary and square integrable in probability, so that the above argument fails. Dimension $d=2$ is critical, and a logarithmic correction is to be expected.

Let us now turn to the weak norm. We further particularize the example by considering the case of vanishing ellipticity ratio, that is, $A \equiv \mathrm{Id}$, and $B_{\varepsilon} \ll 1$ is a stochastic perturbation such that $\left\langle B_{\varepsilon}\right\rangle=0$. This has already been of some help in [7] to identify relevant scalings. In this regime, we decompose $u_{\varepsilon}$ as $u_{\varepsilon}=u_{0}+w_{\varepsilon}$, where $u_{0}$ is deterministic and solves

$$
-\triangle u_{0}=f \text {, }
$$


and $w_{\varepsilon}$ fluctuates and is given by

$$
-\triangle w_{\varepsilon}=\nabla \cdot B_{\varepsilon} \nabla\left(u_{0}+w_{\varepsilon}\right) \simeq \nabla \cdot B_{\varepsilon} \nabla u_{0},
$$

at first order. Denoting by $G$ the Green's function of the Laplace operator on $D$ with homogeneous Dirichlet boundary conditions, we may write $w_{\varepsilon}$ as

$$
w_{\varepsilon}(x) \simeq \int_{D} \nabla G(x, y) \cdot B_{\varepsilon}(y) \nabla u_{0}(y) d y
$$

so that $\mathcal{F}_{w}$ takes the form

$$
\begin{aligned}
\mathcal{F}_{w}(\varepsilon, g) & \simeq\left\langle\left(\int_{D} g(x) w_{\varepsilon}(x) d x\right)^{2}\right\rangle \\
& =\left\langle\left(\int_{D} g(x) \int_{D} \nabla G(x, y) \cdot B_{\varepsilon}(y) \nabla u_{0}(y) d y d x\right)^{2}\right\rangle .
\end{aligned}
$$

Expanding the square and recalling that the correlation length of the perturbation $B_{\varepsilon}$ is $\varepsilon$, this turns into

$$
\begin{array}{r}
\mathcal{F}_{w}(\varepsilon, g) \lesssim \int_{D} \int_{D}|g(x)|\left|g\left(x^{\prime}\right)\right| \int_{D} \int_{D,\left|y-y^{\prime}\right| \leq \varepsilon}|\nabla G(x, y)|\left|\nabla G\left(x^{\prime}, y^{\prime}\right)\right| \\
\times\left|\nabla u_{0}(y)\right|\left|\nabla u_{0}\left(y^{\prime}\right)\right| d y d y^{\prime} d x d x^{\prime},
\end{array}
$$

where $\lesssim$ stands for $\leq$ up to a constant depending only on $\alpha, \beta$, and $d$. Appealing then to the "explicit" formula for the Green function of the Laplace equation (for $d>2$ )

$$
G(x, z)=C_{d} \frac{1}{|x-z|^{d-2}}+g(x, z),
$$

where $g$ is a harmonic (and hence smooth) function, and "discarding the singularity at zero" (the argument can be made rigorous), we then deduce that

$$
\mathcal{F}_{w}(\varepsilon, g) \lesssim \varepsilon^{d}\|g\|_{L^{2}(D)}^{2}\left\|\nabla u_{0}\right\|_{L^{2}(D)}^{2},
$$

which is the central limit theorem scaling.

Our first result is the following (suboptimal) estimates for the strong and weak measures of the fluctuation: There exists a Hölder exponent $0<\gamma<1$ depending only on the ellipticity ratio $\beta / \alpha$ and on $d$ (and which goes to 1 as $\beta / \alpha \rightarrow 1$ ) such that

$$
\begin{gathered}
\mathcal{F}_{s}(\varepsilon) \lesssim\|f\|_{L^{2}(D)}^{2}\left\{\begin{array}{lll}
d=2 & : \varepsilon^{2 \gamma}, \\
d=3 & : \varepsilon^{1+\min \{1,2 \gamma\}}, \\
d>3 & : \varepsilon^{2},
\end{array}\right. \\
\mathcal{F}_{w}(\varepsilon, g) \lesssim\|f\|_{L^{2}(D)}^{2}\|g\|_{L^{\infty}(D)}^{2} \varepsilon^{d-2(1-\gamma)} .
\end{gathered}
$$

In particular, only the estimate of the $L^{2}$-norm of the variance for $d>3$ is optimal. The other estimates are suboptimal and are asymptotically optimal as the ellipticity ratio $\beta / \alpha$ goes to 1 .

Our proof makes use of tools developed by Otto and the author in a series of papers dedicated to quantitative estimates in stochastic homogenization. In $[7,8,6]$, the emphasis was essentially put on the corrector equation and on error estimates for approximations of the homogenized coefficients. In the present work, we adopt a somewhat different point of view: We do not address the convergence of $u_{\varepsilon}$ towards the solution of the homogenized 
problem at the first place, but rather investigate the statistics of $u_{\varepsilon}$ when the correlation length of the noise becomes small - independently of homogenization properties. In particular, we estimate how much the solution $u_{\varepsilon}$ fluctuates around its expectation $\left\langle u_{\varepsilon}\right\rangle$ in terms of the correlation length $\varepsilon$. To this aim, we focus on the weak and strong measures of the fluctuation, $\mathcal{F}_{s}(\varepsilon)$ and $\mathcal{F}_{w}(g, \varepsilon)$. The key ingredient in our proofs is the general variance estimate of [6, Lemma 1.3] (see Lemma 2.1 below), that we apply to $u_{\varepsilon}(x)$ and to $\int_{D} u_{\varepsilon}(x) g(x) d x$, and combine with optimal (and suboptimal) decay estimates for the Green's function and its gradient.

A further question of interest for practical purposes (in particular in the context of identification of diffusion coefficients in presence of noise, see for instance [14]) is the validity of a central limit theorem for the random variable $\int_{D} g(x)\left(u_{\varepsilon}(x)-\left\langle u_{\varepsilon}(x)\right\rangle\right) d x$, which is only known to hold in dimension one (see [1]). Estimate (1.7) is a first piece of answer in dimension $d \geq 2$. Yet this estimate is still too weak. In particular we expect the variance of this quantity to be bounded by $\varepsilon^{d}$, so that a natural question, and next step, would be to prove that the rescaled quantity $\varepsilon^{-d / 2}\left(\int_{D} g(x)\left(u_{\varepsilon}(x)-\left\langle u_{\varepsilon}(x)\right\rangle\right) d x\right)$ converges in law to a centered Gaussian random variable. With this in mind we provide a second result for which the weak estimate is optimal for $\beta / \alpha$ close to 1 (and not only asymptotically optimal). Before we state it, let us mention some earlier work on the subject.

In the case when the perturbation is in the zero-order term, that is for $u_{\varepsilon} \in H^{1}\left(\mathbb{R}^{d}\right)$ solution in $\mathbb{R}^{d}$ to

$$
\left(1+b_{\varepsilon}\right) u_{\varepsilon}-\nabla \cdot A \nabla u_{\varepsilon}=f
$$

for some scalar noise $b_{\varepsilon}$, the problem has been solved for $d \geq 4$ by Figari, Orlandi \& Papanicolaou in [3], and more recently for $d \leq 3$ by Bal in [1]. In their works, they also provide a precise description of the limit law, which we don't do in this article.

In the case of interest here, i. e. when the noise is in the diffusion coefficient, let us mention the very insightful contributions by Yurinskil for continuous elliptic equations, and by Conlon \& Naddaf for discrete elliptic equations. In [16, Theorem 3.1], Yurinskiu essentially proves the algebraic decay (with some small but positive exponent) of some norm of the difference between $u_{\varepsilon}$ and the first two terms of the expansion (1.3). In [2], Conlon \& Naddaf have addressed the problem under investigation here in the discrete setting. They consider the discrete elliptic equation on $\varepsilon \mathbb{Z}^{d}$ (see Subsection 2.2 for precise notation)

$$
u_{\varepsilon}-\nabla_{\varepsilon}^{*} \cdot\left(\mathrm{Id}+B_{\varepsilon}\right) \nabla_{\varepsilon} u_{\varepsilon}=f
$$

and have proved the following bounds on the corresponding version of $\mathcal{F}_{s}$ and $\mathcal{F}_{w}$ (see $[2$, Theorems $1.2 \& 1.3]$ ): There exists $0<\gamma \leq 2$ depending only on $\alpha, \beta$, and $d$ such that

$$
\mathcal{F}_{s}(\varepsilon) \lesssim \varepsilon^{\gamma} \text {. }
$$

In addition, for $d=2, \gamma$ can be chosen arbitrary close to 2 provided $\beta / \alpha$ is taken sufficiently close to 1 , whereas for $d \geq 3, \gamma=2$ for $\beta / \alpha$ close to 1 . For the weak norm, they have proved that there exists $0<\gamma \leq d$ depending only on $\alpha, \beta$ and $d$ such that

$$
\mathcal{F}_{w}(\varepsilon, g) \lesssim \varepsilon^{\gamma} .
$$

In addition, $\gamma$ can be taken arbitrarily close to $d$ if $\beta / \alpha$ is taken sufficiently close to 1 . The proof by Conlon \& Naddaf is rather intricate and makes use of some ideas by Papanicolaou \& Varadhan in [15], an integrability result with respect to a Walsh decomposition of the probability space $L^{2}(\Omega)$ associated with the noise (obtained by singular integrals 
arguments), and a suitable Fourier decomposition. In this paper, we shall slightly improve Conlon \& Naddaf's results (see Theorem 2). In particular, when the problem is posed on the whole space (that is $\varepsilon \mathbb{Z}^{d}$ and not $D \cap \varepsilon \mathbb{Z}^{d}$ ) and when $A$ is constant, the Green's function is stationary. This property can be used to further benefit from the decay of gradients of the Green's function even when the diffusion coefficient is discrete (or simply measurable in the continuum case). We shall indeed improve estimates (1.6) and (1.7) the following way: In addition to the Hölder exponent $0<\gamma \leq 1$, there exists a Meyers exponent $p>2$ (that is a higher integrability exponent, see [12]) depending only on the ellipticity ratio $\beta / \alpha$ and on $d$ (and which goes to infinity when the ratio tends to 1 ) such that

$$
\begin{aligned}
& \mathcal{F}_{s}(\varepsilon) \lesssim\|f\|_{L^{2}\left(\mathbb{R}^{d}\right)}^{2} \begin{cases}d=2 & : \varepsilon^{2} \max \left\{|\ln \varepsilon|,|\ln \varepsilon|^{\max \{0,4-p\}} \varepsilon^{-(1-\gamma) \max \{0,4-p\}}\right\}, \\
d=3: \begin{cases}\varepsilon^{2} \varepsilon^{p-3+\frac{\gamma}{1-\gamma}} & \text { for } p<3-\frac{\gamma}{1-\gamma}, \\
\varepsilon^{2}|\ln \varepsilon| & \text { for } p=3-\frac{\gamma}{1-\gamma}, \\
\varepsilon^{2}\end{cases} \end{cases} \\
& \begin{cases}\varepsilon^{2} & \text { for } p>3-\frac{\gamma}{1-\gamma},\end{cases} \\
& \mathcal{F}_{w}(\varepsilon, g) \lesssim\|f\|_{L^{2}\left(\mathbb{R}^{d}\right)}^{2}\|g\|_{L^{\infty}\left(\mathbb{R}^{d}\right)}^{2} \varepsilon^{d-(1-\gamma) \max \{0,4-p\}} \begin{cases}d=2: & :\left.\ln \varepsilon\right|^{\max \{0,4-p\}} \\
d>2 & : 1 .\end{cases}
\end{aligned}
$$

In particular, for $d>3$ the scaling of $\mathcal{F}_{s}$ is optimal no matter what $\beta / \alpha$, whereas for $d \leq 3$ the scaling of $\mathcal{F}_{s}$ is optimal for ellipticity ratios close to 1 (at least those such that $p \geq 4$ ), as well for $\mathcal{F}_{w}$ for all $d \geq 2$. In addition, for $d>2$, (1.11) \& (1.12) provide upper bounds which are independent of the ellipticity ratio (thus improving (1.9) and (1.10)). Note that we also precisely identify the logarithmic correction for $\mathcal{F}_{s}$ in dimension $d=2$. In the continuum case on the whole space (that is $\mathbb{R}^{d}$ in place of $D$ ), (1.11) \& (1.12) improve estimates (1.6) \& (1.7) in the case when $A$ is constant (see discussion at the end of Subsection 2.2). For ellipticity ratios $\beta / \alpha$ close to $1,(1.12)$ is optimal, which is a first step towards the analysis of $\varepsilon^{-d / 2}\left(\int_{\mathbb{R}^{d}} g(x)\left(u_{\varepsilon}(x)-\left\langle u_{\varepsilon}(x)\right\rangle\right) d x\right)$.

The article is organized as follows. In Section 2, we state the main results of this paper - the estimates of $\mathcal{F}_{s}$ and $\mathcal{F}_{w}$. We first address the problem on a bounded domain in the continuum case (see in particular [6]). We then turn to the case of unbounded domains, and detail the discrete case on $\varepsilon \mathbb{Z}^{d}$ in the simplest possible setting, that is in the case of a discrete elliptic equation with independent and identically distributed (i. i. d.) random conductivities, as Conlon \& Naddaf in [2], and Otto and the author in [7, 8]. Section 3 is dedicated to the proofs of the results on a bounded domain, whereas Section 4 deals with the discrete case on $\varepsilon \mathbb{Z}^{d}$.

Throughout the paper, we make use of the following notation:

- $d \geq 2$ is the dimension;

- in the discrete case, for all $\varepsilon>0, \int_{\varepsilon \mathbb{Z}^{d}} d x$ denotes the sum over $x \in \mathbb{Z}^{d}$ times the measure $\varepsilon^{d}$;

- $\langle\cdot\rangle$ is the ensemble average, or equivalently the expectation in the underlying probability space;

- $\operatorname{var}[\cdot]$ and $\operatorname{cov}[\cdot ; \cdot]$ are the variance and covariance associated with the ensemble average, respectively; 
- $\lesssim$ and $\gtrsim$ stand for $\leq$ and $\geq$ up to a multiplicative constant which only depends on the dimension $d$ and the constants $\alpha, \beta$ (see Definition 2.3 below) if not otherwise stated;

- when both $\lesssim$ and $\gtrsim$ hold, we simply write $\sim$;

- we use $\gg$ instead of $\gtrsim$ when the multiplicative constant is (much) larger than 1 ;

- $\left(\mathbf{e}_{1}, \ldots, \mathbf{e}_{d}\right)$ denotes the canonical basis of $\mathbb{R}^{d}$;

- $|\cdot|$ denotes the Euclidean norm in $\mathbb{R}^{d},|\cdot|_{\infty}$ denotes the supremum norm in $\mathbb{R}^{d}$.

\section{Statement of the main Results}

2.1. The bounded domain case. We first introduce the notion of conductivity function.

Definition 2.1. We denote by $\mathcal{A}_{\alpha \beta}$ the set of measurable functions on $\mathbb{R}^{d}$ taking values in the set of symmetric $d \times d$-matrices with ellipticity constants $\beta \geq \alpha>0$ : For all $A \in \mathcal{A}_{\alpha \beta}$, almost every $x \in \mathbb{R}^{d}$, and all $\xi \in \mathbb{R}^{d}$,

$$
\xi \cdot A(x) \xi \geq \alpha|\xi|^{2}, \quad|A(x) \xi| \leq \beta|\xi| .
$$

We now turn to the definition of the statistics of the noise.

Definition 2.2. A random noise $B \in \mathcal{A}_{\alpha \beta}$ is said to be admissible if it is stationary in the sense that for almost every $y \in \mathbb{R}^{d}, k \in \mathbb{N}$ and $\left(y_{0}, \ldots, y_{k}\right) \in\left(\mathbb{R}^{d}\right)^{k+1},\left(B\left(y_{0}+\right.\right.$ $\left.\cdot), \ldots, B\left(y_{k}+\cdot\right)\right)$ and $\left(B\left(y_{0}+y+\cdot\right), \ldots, B\left(y_{k}+y+\cdot\right)\right)$ have the same statistics. It is of finite correlation length if there exists $C_{L} \in \mathbb{R}^{+}$such that for all $x, y \in \mathbb{R}^{d}, B(x)$ and $B(y)$ are independent for $|x-y| \geq C_{L}$.

Note that this "definition" of the correlation-length is not standard. The main result in the continuum case is as follows.

Theorem 1. Let $D$ be a bounded domain of $\mathbb{R}^{d}$ which satisfies a uniform exterior cone condition. Let $A$ be a conductivity function on $D$, and $B$ be an admissible random noise of finite correlation length such that $A(x)+B(y) \in \mathcal{A}_{\alpha \beta}$ for almost all $x \in D, y \in \mathbb{R}^{d}$. Let $f \in L^{2}(D)$, and for all $\varepsilon>0$, let $u_{\varepsilon} \in H_{0}^{1}(D)$ denote the unique weak solution to

$$
-\nabla \cdot\left(A+B_{\varepsilon}\right) \nabla u_{\varepsilon}=f, \quad \text { in } D,
$$

where $B_{\varepsilon}(\cdot):=B(\cdot / \varepsilon)$.

Then, there exists a Hölder exponent $0<\gamma \leq 1$ depending only on $\alpha, \beta$, and $d$ (and which tends to 1 when $\beta / \alpha \rightarrow 1$ ) such that for all $g \in L^{\infty}(D)$, the fluctuation of $u_{\varepsilon}$ is estimated by:

$$
\begin{aligned}
& \int_{D} \operatorname{var}\left[u_{\varepsilon}(x)\right] d x \lesssim\|f\|_{2}^{2}\left\{\begin{array}{lll}
d=2 & : & \varepsilon^{2 \gamma}, \\
d=3 & : & \varepsilon^{1+\min \{1,2 \gamma\}}, \\
d>3 & : & \varepsilon^{2},
\end{array}\right. \\
& \operatorname{var}\left[\int_{D} g(x) u_{\varepsilon}(x) d x\right] \lesssim\|f\|_{2}^{2}\|g\|_{\infty}^{2} \varepsilon^{d-2(1-\gamma)} \text {. }
\end{aligned}
$$


2.2. The unbounded domain case. In order to make the comparison to Conlon \& Naddaf's result, we present the discrete case in detail. The notions of discrete conductivity function and elliptic operator are as follow.

Definition 2.3. We say that $a$ is a conductivity function if there exist $0<\alpha \leq \beta<\infty$ such that for every edge $e=(x, y)$ (with $x, y \in \mathbb{Z}^{d},|x-y|=1$ ), one has $a(e) \in[\alpha, \beta]$. We denote by $\mathcal{A}_{\alpha \beta}$ the set of such conductivity functions.

Definition 2.4. For all $\varepsilon>0$, the elliptic operator $L_{\varepsilon}: L_{\mathrm{loc}}^{2}\left(\varepsilon \mathbb{Z}^{d}\right) \rightarrow L_{\mathrm{loc}}^{2}\left(\varepsilon \mathbb{Z}^{d}\right), u \mapsto L_{\varepsilon} u$ associated with a conductivity function $a \in \mathcal{A}_{\alpha \beta}$ is defined for all $x \in \varepsilon \mathbb{Z}^{d}$ by

$$
\left(L_{\varepsilon} u\right)(x)=-\nabla_{\varepsilon}^{*} \cdot A_{\varepsilon}(x) \nabla_{\varepsilon} u(x)
$$

where

$$
\nabla_{\varepsilon} u(x):=\varepsilon^{-1}\left[\begin{array}{l}
u\left(x+\varepsilon \mathbf{e}_{1}\right)-u(x) \\
\vdots \\
u\left(x+\varepsilon \mathbf{e}_{d}\right)-u(x)
\end{array}\right], \nabla_{\varepsilon}^{*} u(x):=\varepsilon^{-1}\left[\begin{array}{l}
u(x)-u\left(x-\varepsilon \mathbf{e}_{1}\right) \\
\vdots \\
u(x)-u\left(x-\varepsilon \mathbf{e}_{d}\right)
\end{array}\right],
$$

and

$$
A_{\varepsilon}(x)=A\left(\varepsilon^{-1} x\right):=\operatorname{diag}\left[a\left(e_{1}\right), \ldots, a\left(e_{d}\right)\right],
$$

$e_{1}=\left[\varepsilon^{-1} x, \varepsilon^{-1} x+\mathbf{e}_{1}\right], \ldots, e_{d}=\left[\varepsilon^{-1} x, \varepsilon^{-1} x+\mathbf{e}_{d}\right]$. In what follows, we will abusively denote by conductivity function both $a$ and the associated $A$ for $\varepsilon=1$, and write as well $A \in \mathcal{A}_{\alpha \beta}$.

We now turn to the definition of the statistics of the conductivity function.

Definition 2.5. A conductivity function $B \in \mathcal{A}_{\alpha \beta}$ is said to be independent and identically distributed (i. i. d.) if the coefficients $b(e)$ are i. i. d. random variables.

The main result on the estimate of the fluctuation of solutions to discrete elliptic equations perturbed by an i. i. d. random noise is the following.

Theorem 2. Let a be a constant conductivity function, and $b$ be an i. i. d. conductivity function on $\mathbb{Z}^{d}$ such that $A+B \in \mathcal{A}_{\alpha \beta}$. Let $f \in C^{0}\left(\mathbb{R}^{d}\right) \cap L^{2}\left(\mathbb{R}^{d}\right)$ and $g \in C_{b}^{0}\left(\mathbb{R}^{d}\right) \cap L^{2}\left(\mathbb{R}^{d}\right)$ (that is, continuous, bounded, and square-integrable), and for all $\varepsilon>0$, let $u_{\varepsilon} \in L^{2}\left(\varepsilon \mathbb{Z}^{d}\right)$ denote the unique solution to

$$
u_{\varepsilon}-\nabla_{\varepsilon}^{*} \cdot\left(A+B_{\varepsilon}\right) \nabla_{\varepsilon} u_{\varepsilon}=f, \quad \text { in } \varepsilon \mathbb{Z}^{d} .
$$

Then there exist a Hölder exponent $0<\gamma \leq 1$ and a Meyers exponent $p>2$ depending only on $\alpha, \beta$ and $d$ (the latter goes to infinity when $\beta / \alpha \rightarrow 1$ ), such that the fluctuation of $u_{\varepsilon}$ is estimated by:

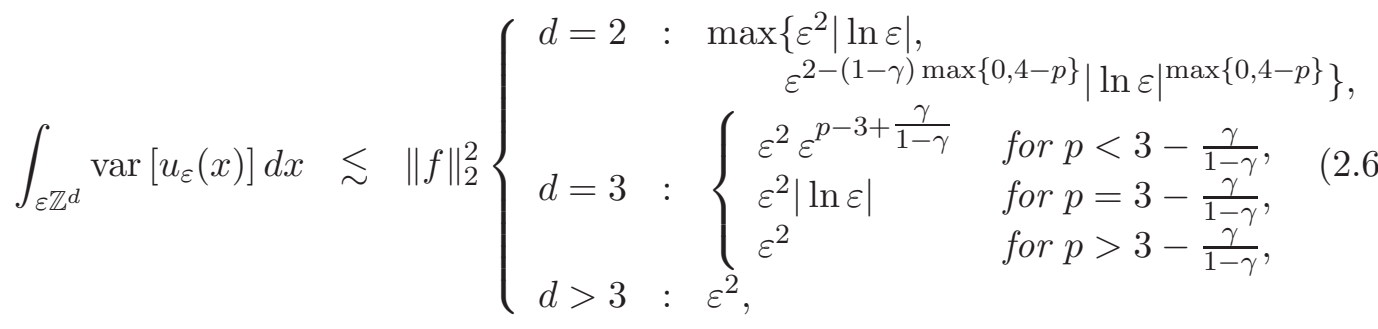

$$
\begin{aligned}
& \operatorname{var}\left[\int_{\varepsilon \mathbb{Z}^{d}} g(x) u_{\varepsilon}(x) d x\right] \lesssim\|f\|_{2}^{2}\|g\|_{\infty}^{2}\left\{\begin{array}{lll}
d=2 & : & \varepsilon^{d-(1-\gamma) \max \{0,4-p\}}|\ln \varepsilon|^{\max \{0,4-p\}} \\
d>2 & : & \varepsilon^{d-(1-\gamma) \max \{0,4-p\}}
\end{array}\right.
\end{aligned}
$$


The estimates of Theorem 2 are optimal when the ellipticity ratio is such that the associated Meyers exponent $p$ is (for instance) larger than 4 .

Theorem 2 improves the results of [2, Theorems $1.2 \& 1.3]$ by Conlon \& Naddaf. For the strong measure (2.6) of the fluctuation, we precisely identify the logarithmic correction for $d=2$, provide with an upper bound independent of the ellipticity ratio for $d=3$, and prove an optimal estimate for $d>3$. For the weak measure (2.7) of the fluctuation, the optimal scaling is reached provided $p$ is larger than 4 (whereas the optimal scaling is only met asymptotically in [2, Theorem 1.3]), and for $d>2$ the estimate gives a non-trivial upper bound uniformly in the ellipticity ratio.

A corresponding result holds in the continuum case, and is stronger that Theorem 1 in the case when the conductivity function $A$ is a constant matrix.

Theorem 3. Let $A$ be a symmetric matrix, and $B$ be an admissible noise with finite correlation-length and such that $A+B \in \mathcal{A}_{\alpha \beta}$. Let $f \in L^{2}\left(\mathbb{R}^{d}\right)$, and for all $\varepsilon>0$, let $u_{\varepsilon} \in H^{1}\left(\mathbb{R}^{d}\right)$ denote the unique weak solution to

$$
u_{\varepsilon}-\nabla \cdot\left(A+B_{\varepsilon}\right) \nabla u_{\varepsilon}=f, \quad \text { in } \mathbb{R}^{d},
$$

where $B_{\varepsilon}(\cdot):=B(\cdot / \varepsilon)$.

Then there exist a Hölder exponent $\gamma>0$ and a Meyers exponent $p>2$ depending only on $\alpha, \beta$ and $d$ (and such that $p \rightarrow \infty$ and $\gamma \rightarrow 1$ when $\beta / \alpha \rightarrow 1$ ), such that for all $g \in L^{1}\left(\mathbb{R}^{d}\right) \cap L^{\infty}\left(\mathbb{R}^{d}\right)$ the fluctuation of $u_{\varepsilon}$ is estimated by:

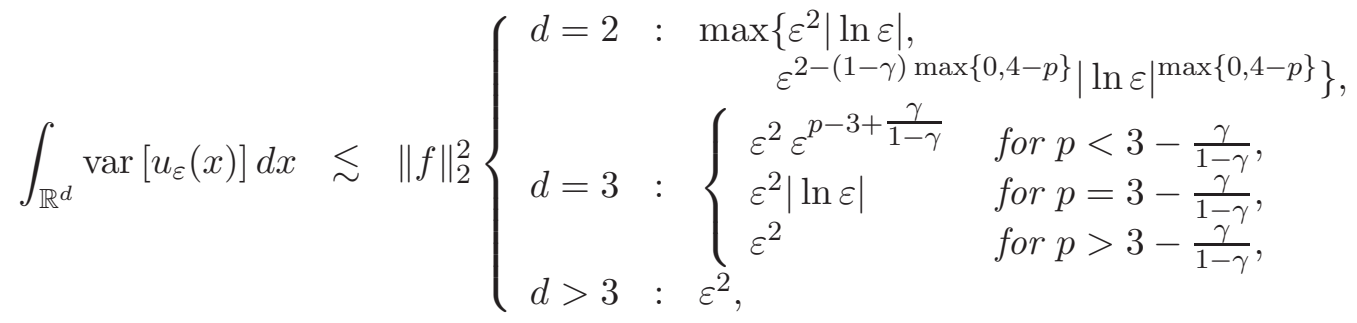

$$
\begin{aligned}
& \operatorname{var}\left[\int_{\mathbb{R}^{d}} g(x) u_{\varepsilon}(x) d x\right] \lesssim\|f\|_{2}^{2}\|g\|_{\infty}^{2}\left\{\begin{array}{lll}
d=2 & : & \varepsilon^{d-(1-\gamma) \max \{0,4-p\}}|\ln \varepsilon|^{\max \{0,4-p\}}, \\
d>2 & : & \varepsilon^{d-(1-\gamma) \max \{0,4-p\}} .
\end{array}\right.
\end{aligned}
$$

The results of this theorem yield stronger bounds than Theorem 1. This is clear for the weak measure of the fluctuation for all $d \geq 2$ and for the strong norm for $d=2$ and $d>3$ since $p>2$. For $d=3$, this is clear for $p \geq 3-\frac{\gamma}{1-\gamma}$, whereas for $p<3-\frac{\gamma}{1-\gamma}$, the result follows from the fact that $\gamma \mapsto p-2+\frac{\gamma}{1-\gamma}-2 \gamma$ is a non-negative function provided $p>2$.

2.3. Structure of the proof in the continuum case on a bounded domain. We begin with the strong norm of the variance. The starting point is the change of variables $x \sim x / \varepsilon$ to make the correlation length be of order 1 :

$$
\int_{D} \operatorname{var}\left[u_{\varepsilon}(x)\right] d x=\varepsilon^{d} \int_{D / \varepsilon} \operatorname{var}\left[v_{\varepsilon}(x)\right] d x
$$

where $v_{\varepsilon}$ is the weak solution in $H_{0}^{1}(D / \varepsilon)$ to

$$
-\nabla \cdot C(x) \nabla v_{\varepsilon}(x)=\varepsilon^{2} f_{\varepsilon}(x)
$$


with $C(x):=A_{\varepsilon}(x)+B(x)$, and

$$
\begin{aligned}
A_{\varepsilon}(x) & =A(\varepsilon x), \\
f_{\varepsilon}(x) & =f(\varepsilon x)
\end{aligned}
$$

and is given for all $x \in D / \varepsilon$ by

$$
v_{\varepsilon}(x)=u_{\varepsilon}(\varepsilon x)
$$

Without loss of generality, we assume the correlation length $C_{L}$ to be less than $1 / 3$, and appeal to the following variance estimate of $[6]$ :

Lemma 2.1 (variance estimate). Let $B$ be random with values in $\mathcal{A}_{\alpha \beta}$ and the following statistical property: The random field $B$ has correlation length unity in the sense that for any two sets $U, V \subset \mathbb{R}^{d}$ with $\operatorname{dist}(U, V) \geq 1 / 3$, the restrictions $B_{\mid U}$ and $B_{\mid V}$ are independent. Let $X$ be a function on $\mathcal{A}_{\alpha \beta}$ - which we consider as a random variable with the following regularity property: $X$ can be uniformly approximated by functions $\tilde{X}$ on $\mathcal{A}_{\alpha \beta}$ that depend on $B$ only through the restriction $B_{\mid \tilde{U}}$ for some compact set $\tilde{U} \subset \mathbb{R}^{d}$. Then we have the following variance estimate

$$
\operatorname{var}[X] \lesssim\left\langle\int_{\mathbb{R}^{d}}\left(\begin{array}{c}
\operatorname{Osc} X \\
B_{\mid Q(z)}
\end{array}\right)^{2} d z\right\rangle,
$$

where osc $X$ denotes the oscillation of $X$ with respect to $B$ restricted onto the cube $Q(z)$ $B_{\mid Q(z)}$

of lateral size unity and center at $z \in \mathbb{R}^{d}$. Note that for any set $U \subset \mathbb{R}^{d},{ }_{B_{\mid U}}^{\operatorname{osc}} X$ itself is a random variable:

$$
\begin{aligned}
& \left(\operatorname{osc}_{B_{\mid U}} X\right)(B)=\left(\sup _{B_{\mid U}} X\right)(B)-\left(\inf _{B_{\mid U}} X\right)(B) \\
& =\sup \left\{X(\tilde{B}) \mid \tilde{B} \in \mathcal{A}_{\alpha \beta}, \quad \tilde{B}_{\mid \mathbb{R}^{d} \backslash U}=B_{\mid \mathbb{R}^{d} \backslash U}\right\} \\
& -\inf \left\{X(\tilde{B}) \mid \tilde{B} \in \mathcal{A}_{\alpha \beta}, \quad \tilde{B}_{\mid \mathbb{R}^{d} \backslash U}=B_{\mid \mathbb{R}^{d} \backslash U}\right\} .
\end{aligned}
$$

Since for all $x \in D / \varepsilon, v_{\varepsilon}(x)$ only depends on the restriction of $B$ onto $D / \varepsilon$, one may apply Lemma 2.1 to $v_{\varepsilon}(x)$, which yields

$$
\operatorname{var}\left[v_{\varepsilon}(x)\right] \lesssim \int_{\mathbb{R}^{d}}\left\langle\begin{array}{c}
\operatorname{OSc}^{2} \\
B_{\mid Q(z)}
\end{array} v_{\varepsilon}(x)\right\rangle d z .
$$

We now estimate the susceptibility of $v_{\varepsilon}(x)$ with respect to the coefficients $B_{\mid Q(z)}$ for some $z \in D / \varepsilon$. To this aim we first define Green's functions:

Definition 2.6 (Green's function). Let $d \geq 2$. The Green's function $G: \mathcal{A}_{\alpha \beta} \times D / \varepsilon \times$ $D / \varepsilon \rightarrow \mathbb{R},(C, x, y) \mapsto G(x, y ; C)$ associated with the conductivity function $C \in \mathcal{A}_{\alpha \beta}$ is defined for all $y \in D / \varepsilon$ as the unique distributional solution in $W_{0}^{1,1}(D / \varepsilon)$ to

$$
-\nabla_{x} \cdot C(x) \nabla_{x} G(x, y ; C)=\delta(y-x) .
$$


In addition, there exists $c>0$ depending only on $\alpha, \beta$, and $d$, such that for all $x, y \in D / \varepsilon$

$$
G(x, y ; C) \lesssim\left\{\begin{array}{lll}
d=2 & : & |\ln (|x-y|)|, \\
d>2 & : & \frac{1}{|x-y|^{d-2}},
\end{array}\right.
$$

and for all $R \lesssim 1$ and $0<\eta<1, x \mapsto G(x, y) \in W^{1, d /(d-1+\eta)}\left(Q_{R}(y)\right)$ uniformly in $y \in D / \varepsilon$ such that $Q_{R}(y) \subset D / \varepsilon$.

Note that $G(x, y ; C)=G(y, x ; C)$ for all $x, y \in D / \varepsilon$, by symmetry of $C$.

Definition 2.6 essentially follows from [11, Theorem (5.1) \& Section 7]. The susceptibility estimate is as follows:

Lemma 2.2 (susceptibility estimate). Let $A$ be a conductivity function on a bounded domain $D \subset \mathbb{R}^{d}$, and $B$ be an admissible random noise of finite correlation length such that $A(x)+B(y) \in \mathcal{A}_{\alpha \beta}$ for almost all $x \in D, y \in \mathbb{R}^{d}$. Let $f \in L^{2}(D)$ and $u_{\varepsilon} \in H_{0}^{1}(D)$ be as in Theorem 1, $G$ be as in Definition 2.6 with $C=A+B$, and $f_{\varepsilon}$ and $v_{\varepsilon}$ be as in (2.10) E (2.11). For notational convenience, we extend $f_{\varepsilon}, v_{\varepsilon}$ and $\nabla v_{\varepsilon}$ by zero on $\mathbb{R}^{d} \backslash D / \varepsilon$, as well as $G$ and $\nabla G$ on $\mathbb{R}^{d} \times \mathbb{R}^{d} \backslash(D / \varepsilon \times D / \varepsilon)$. Then for all $|z-x| \geq 1$, we have

$$
\underset{B_{\mid Q(z)}}{\operatorname{Osc}^{2}} v_{\varepsilon}(x) \lesssim \int_{Q(z)}\left|\nabla_{y} G(x, y)\right|^{2} d y \int_{Q(z)}\left|\nabla v_{\varepsilon}(y)\right|^{2} d y
$$

whereas for all $|x-z|<1$ we have

$$
\underset{B_{\mid Q(z)}}{\operatorname{Osc}^{2}} v_{\varepsilon}(x) \lesssim \int_{Q(z)}\left|\nabla v_{\varepsilon}(y)\right|^{2} d y+\varepsilon^{4} \int_{Q_{4}(z)} f_{\varepsilon}(y)^{2} d y
$$

To prove Lemma 2.2 we first assume that $A+B$ is smooth, and then conclude by approximation using the following lemma.

Lemma 2.3 (regularization). For all $C \in \mathcal{A}_{\alpha \beta}, f_{\varepsilon} \in L^{2}(D / \varepsilon)$ and $h>0$, let $C_{h}$ be defined for all $x \in D / \varepsilon$ by

$$
\begin{aligned}
C_{h}(x) & =\int_{D / \varepsilon} \rho_{h}(x-y) C(y) d y, \\
f_{\varepsilon}^{h}(x) & =\int_{D / \varepsilon} \rho_{h}(x-y) f_{\varepsilon}(y) d y,
\end{aligned}
$$

where $\rho_{h}(y)=h^{-d} \rho(y / h)$, and $\rho$ is a smooth non-negative function on $\mathbb{R}^{d}$ of unit mass with compact support containing the origin. Note that for all $h>0$ there exists $\alpha_{h}>0$ (which goes to $\alpha$ as $h$ vanishes) such that $C_{h} \in \mathcal{A}_{\alpha_{h} \beta} \cap C^{\infty}(D / \varepsilon)$, and $f_{\varepsilon}^{h} \in C^{\infty}(D / \varepsilon)$. Denoting by $G$ and $v_{\varepsilon}$, and $G^{h}$ and $v_{\varepsilon}^{h}$ the Green's functions and solution to (2.9) associated with $C$ and $C_{h}$, and $f_{\varepsilon}$ and $f_{\varepsilon, h}$, respectively, we have:

(a) For all $y \in D / \varepsilon, G^{h}(\cdot, y)$ converges to $G(\cdot, y)$ in $L^{1}(D / \varepsilon)$, and in $L^{q}\left(D / \varepsilon \backslash B_{r}(y)\right)$ and $H^{1}\left(D / \varepsilon \backslash B_{r}(y)\right)$ for all $1 \leq q<\infty$ and $r>0$;

(b) The function $v_{\varepsilon}^{h}$ converges pointwise everywhere on $D / \varepsilon$ and in $H^{1}(D / \varepsilon)$ to $v_{\varepsilon}$.

In view of Lemma 2.2 we shall need to estimate gradients of the Green's function: 
Lemma 2.4. Let $D$ satisfy a uniform exterior cone condition. Then there exists $0<\gamma \leq 1$ depending only on $\alpha, \beta$, and d such that for all $G: \mathcal{A}_{\alpha \beta} \times D / \varepsilon \times D / \varepsilon \rightarrow \mathbb{R},(C, x, y) \mapsto$ $G(x, y ; C)$, and all $x, y \in D / \varepsilon$ with $|y-x| \gg 1$ we have

$$
\int_{Q(x) \cap D / \varepsilon}\left|\nabla_{x^{\prime}} G\left(x^{\prime}, y ; C\right)\right| d x^{\prime} \lesssim\left\{\begin{array}{lll}
d=2 & : & \frac{|\ln (|x-y|)|}{|x-y|^{\gamma}} \\
d>2 & : & \frac{1}{|x-y|^{d-2+\gamma}}
\end{array}\right.
$$

Note that the symmetry assumption on the coefficients $A$ and $B$ is not necessary (at least for $d>2$ ) since [9, (1.9) Theorem] holds as well for non-symmetric coefficients.

The proof of the estimate of the strong norm of the variance then follows from the combination of (2.14), (2.17), (2.18), and (2.19).

We now turn to the estimate of the weak norm of the variance, which we rewrite as

$$
\operatorname{var}\left[\int_{D} u_{\varepsilon}(x) g(x) d x\right]=\varepsilon^{2 d} \operatorname{var}\left[\int_{D / \varepsilon} v_{\varepsilon}(x) g_{\varepsilon}(x) d x\right],
$$

where $g_{\varepsilon}(x):=g(\varepsilon x)$ for all $x \in D$. Since $B \mapsto \int_{D} u_{\varepsilon}(x) g(x) d x$ only depends on the restriction of $B$ onto $D / \varepsilon$, one may apply the variance estimate, so that

$$
\operatorname{var}\left[\int_{D} u_{\varepsilon}(x) g(x) d x\right] \lesssim \varepsilon^{2 d}\left\langle\int_{\mathbb{R}^{d}} \underset{B_{\mid Q(z)}}{\operatorname{Osc}^{2}}\left(\int_{D / \varepsilon} v_{\varepsilon}(x) g_{\varepsilon}(x) d x\right) d z\right\rangle .
$$

Since $g_{\varepsilon}$ does not depend on $B$, we may use the elementary inequality

$$
\begin{aligned}
\underset{B_{\mid Q(z)} \operatorname{osc}}{\operatorname{osc}}\left(\int_{D / \varepsilon} v_{\varepsilon}(x) g_{\varepsilon}(x) d x\right) & \leq \int_{D / \varepsilon} B_{\mid Q(z)}^{\operatorname{Osc}}\left(v_{\varepsilon}(x) g_{\varepsilon}(x)\right) d x \\
& \leq \int_{D / \varepsilon}\left(\begin{array}{cc}
\operatorname{Osc} & \left.v_{\varepsilon}(x)\right) \\
B_{\mid Q(z)}
\end{array}\left|g_{\varepsilon}(x)\right| d x\right.
\end{aligned}
$$

which turns the variance estimate into

$$
\operatorname{var}\left[\int_{D} u_{\varepsilon}(x) g(x) d x\right] \lesssim \varepsilon^{2 d} \int_{\mathbb{R}^{d}}\left\langle\left[\int_{D / \varepsilon}\left(\begin{array}{c}
\operatorname{Osc} \\
B_{\mid Q(z)}
\end{array} v_{\varepsilon}(x)\right)\left|g_{\varepsilon}(x)\right| d x\right]^{2}\right\rangle d z .
$$

The weak estimate will follow from the combination of (2.20) with (2.17), (2.18), and (2.19).

2.4. Structure of the proof in the discrete case on $\varepsilon \mathbb{Z}^{d}$. The proof of Theorem 2 slightly departs from the proof of Theorem 3. In the case of an unbounded domain (and a constant matrix $A$ ) the associated Green's function is stationary (see Definition 2.8 below), so that

$$
\left\langle\nabla_{x} G(x, y)\right\rangle=\left\langle\nabla_{x} G(x-y, 0)\right\rangle=-\left\langle\nabla_{y} G(x-y, 0)\right\rangle=-\left\langle\nabla_{y} G(x, y)\right\rangle .
$$

This allows to replace a derivative in one variable by a derivative in the other variable, which - as we shall see in the proof - enables us to benefit from the better decay of the gradient of the Green's function when integrated on dyadic annuli (see Lemma 2.8). Yet, in order to make this strategy work, the starting point is slightly different from the 
previous subsection, and we first appeal to the following Green representation formula for $u_{\varepsilon}$ :

$$
u_{\varepsilon}(\varepsilon x)=\varepsilon^{2} \int_{\mathbb{Z}^{d}} G_{\varepsilon^{2}}(x, y) f(\varepsilon y) d y,
$$

where the Green's function $G_{\varepsilon^{2}}$ is defined as follows:

Definition 2.7 (discrete Green's function). Let $d \geq 2$. For all $\eta>0$, the Green's function $G_{\eta}: \mathcal{A}_{\alpha \beta} \times \mathbb{Z}^{d} \times \mathbb{Z}^{d} \rightarrow \mathbb{Z}^{d},(c, x, y) \mapsto G_{\eta}(x, y ; c)$ associated with the conductivity function $c \in \mathcal{A}_{\alpha \beta}$ is defined for all $y \in \mathbb{Z}^{d}$ as the unique solution in $L_{x}^{2}\left(\mathbb{Z}^{d}\right)$ to

$$
\int_{\mathbb{Z}^{d}} \eta G_{\eta}(x, y ; a) v(x) d x+\int_{\mathbb{Z}^{d}} \nabla v(x) \cdot C(x) \nabla_{x} G_{\eta}(x, y ; a) d x=v(y), \quad \forall v \in L^{2}\left(\mathbb{Z}^{d}\right),
$$

where $C$ is as in (2.4) with $c$ in place of $a$.

Definition 2.8. The conductivity matrix $A+B$ is stationary in the sense that for all $k \in \mathbb{N}$, and all $z, z_{1}, \ldots, z_{k} \in \mathbb{Z}^{d},\left(A+B\left(\cdot+z_{1}\right), \ldots, A+B\left(\cdot+z_{k}\right)\right)$ and $(A+B(\cdot+z+$ $\left.\left.z_{1}\right), \ldots, A+B\left(\cdot+z+z_{k}\right)\right)$ have the same statistics, so that for all $x, z \in \mathbb{Z}^{d}$,

$$
\langle A+B(x+z)\rangle=\langle A+B(x)\rangle .
$$

Any translation invariant function of $A+B$, such as the Green's functions $G_{\eta}$ of Definition 2.7, is jointly stationary with $A+B$. In this case, stationarity implies that $G_{\eta}(\cdot+z, \cdot+z)$ has the same statistics as $G_{\eta}(\cdot, \cdot)$ for all $z \in \mathbb{Z}^{d}$, so that in particular, for all $x, y, z \in \mathbb{Z}^{d}$,

$$
\left\langle G_{\eta}(x+z, y+z)\right\rangle=\left\langle G_{\eta}(x, y)\right\rangle .
$$

We may rewrite the strong norm of the fluctuation of $u_{\varepsilon}$ in the form

$$
\begin{aligned}
\int_{\varepsilon \mathbb{Z}^{d}} \operatorname{var}\left[u_{\varepsilon}(x)\right] d x & =\left\langle\varepsilon^{d} \int_{\mathbb{Z}^{d}}\left(\varepsilon^{2} \int_{\mathbb{Z}^{d}}\left(G_{\varepsilon^{2}}(x, y)-\left\langle G_{\varepsilon^{2}}(x, y)\right\rangle\right) f(\varepsilon y) d y\right)^{2} d x\right\rangle \\
& =\varepsilon^{d+4} \int_{\mathbb{Z}^{d}} \int_{\mathbb{Z}^{d}} \int_{\mathbb{Z}^{d}} f(\varepsilon y) f\left(\varepsilon y^{\prime}\right) \operatorname{cov}\left[G_{\varepsilon^{2}}(x, y) ; G_{\varepsilon^{2}}\left(x, y^{\prime}\right)\right] d y d y^{\prime} d x .
\end{aligned}
$$

We thus need to control not a variance, but rather a covariance (of the Green's function). To this aim we recall the following stronger form of the spectral gap estimate, which is the desired covariance estimate:

Lemma 2.5 (covariance estimate). [8, Lemma 3] Let $a=\left\{a_{i}\right\}_{i \in \mathbb{N}}$ be a sequence of $i$. $i$. $d$. random variables with range $[\alpha, \beta]$. Let $X$ and $Y$ be two Borel measurable functions of $a \in \mathbb{R}^{\mathbb{N}}$ (i. e. measurable w. r. t. the smallest $\sigma$-algebra on $\mathbb{R}^{\mathbb{N}}$ for which all coordinate functions $\mathbb{R}^{\mathbb{N}} \ni a \mapsto a_{i} \in \mathbb{R}$ are Borel measurable, $c f$. [10, Definition 14.4]). Then we have

$$
\operatorname{cov}[X ; Y] \leq \sum_{i=1}^{\infty}\left\langle\sup _{a_{i}}\left|\frac{\partial X}{\partial a_{i}}\right|^{2}\right\rangle^{1 / 2}\left\langle\sup _{a_{i}}\left|\frac{\partial Y}{\partial a_{i}}\right|^{2}\right\rangle^{1 / 2} \operatorname{var}\left[a_{1}\right]
$$

where $\sup _{a_{i}}\left|\frac{\partial Z}{\partial a_{i}}\right|$ denotes the supremum of the modulus of the $i$-th partial derivative

$$
\frac{\partial Z}{\partial a_{i}}\left(a_{1}, \cdots, a_{i-1}, a_{i}, a_{i+1}, \cdots\right)
$$

of $Z$ with respect to the variable $a_{i} \in[\alpha, \beta]$, for $Z=X, Y$. 
In order to apply Lemma 2.5 to the Green's function, one needs to know that it satisfies the required measurability assumption, which is the object of the following lemma proved in [7].

Lemma 2.6. [7, Lemma 2.6] Let $c \in \mathcal{A}_{\alpha \beta}$ be an i. $i$. d. conductivity function, and let $G_{\eta}(\cdot, \cdot ; c)$ be the associated Green's function for $\eta>0$. Then for fixed $x, y \in \mathbb{Z}^{d}, G_{\eta}(x, y, \cdot)$ is continuous w. r. t. the product topology of $\mathcal{A}_{\alpha \beta}$ (i. e. the smallest/coarsest topology on $\mathbb{R}^{E}$, where $\mathbb{B}$ denotes the set of edges, such that the coordinate functions $\mathbb{R}^{E} \ni c \mapsto c_{e} \in \mathbb{R}$ are continuous for all edges $e \in \mathbb{B}$ ).

In particular, $G_{\eta}(x, y ; \cdot)$ is a Borel measurable function of $c \in \mathcal{A}_{\alpha \beta}$, so that one may apply Lemma 2.5 to $G_{\eta}(x, y ; \cdot)$ and nonlinear funtions thereof.

In order to exploit Lemma 2.5, we appeal to the estimate derived in [7] on the susceptibility of the Green's function with respect to the conductivity function:

Lemma 2.7. [7, Lemma 2.5] Let $G_{\eta}: \mathcal{A}_{\alpha \beta} \times \mathbb{Z}^{d} \times \mathbb{Z}^{d} \rightarrow \mathbb{R},(c, x, y) \mapsto G_{\eta}(x, y ; c)$ be the Green's function associated with the conductivity function c for $\eta>0$. For all $e=\left[z, z+\mathbf{e}_{i}\right]$ and for all $x, y \in \mathbb{Z}^{d}$, it holds that

$$
\frac{\partial}{\partial c(e)} G_{\eta}(x, y ; c)=-\nabla_{z_{i}} G_{\eta}(x, z ; c) \nabla_{z_{i}} G_{\eta}(z, y ; c) .
$$

As a by-product we also have: For all $x \in \mathbb{Z}^{d}$

$$
\sup _{c(e)}\left|\nabla_{z_{i}} G_{\eta}(z, x ; c)\right| \lesssim\left|\nabla_{z_{i}} G_{\eta}(z, x ; c)\right| .
$$

Once we combine the covariance estimate of $G_{\eta}$ with (2.22) and Lemma 2.7, it only remains to estimate the integrals involving gradients of the Green's function. As will be clear in the proof, the exponents we obtain in the estimates for the strong and weak norms would be optimal if we knew that $\nabla_{y} G_{\eta}(x, y)$ had the optimal decay $(1+|x-y|)^{1-d} \exp (-c|x-y| \sqrt{\eta})$ for some $c>0$. This estimate cannot hold pointwise uniformly with respect to the ellipticity ratio. Yet, using Cacciopoli's inequality, it survives for the square of the gradient integrated on (dyadic) annuli. We will actually need more, and will use that the averaged optimal decay also holds for a higher power than 2 - depending on the ellipticity ratio. This is a consequence of the following Meyers estimate.

Lemma 2.8 (higher integrability of gradients). Let $a \in \mathcal{A}_{\alpha \beta}$ be a conductivity function, and $G_{\eta}$ be its associated Green's function. Then, for $d \geq 2$, there exist $p>2$ and $c>0$ depending only on $\alpha, \beta$, and $d$ such that for all $\eta>0, p \geq q \geq 2, k>0$ and $R \gg 1$,

$$
\int_{R<|z| \leq 2 R}\left|\nabla_{z} G_{\eta}(z, 0)\right|^{q} d z \lesssim R^{d}\left(R^{1-d}\right)^{q} \exp (-c q R \sqrt{\eta}) .
$$

Lemma 2.8 is direct consequence of [7, Lemma 2.9], and [5, Lemma 2]. For technical reasons, at some places we will have no choice but estimate $\nabla G_{\eta}(x, y)$ pointwise. To this aim we use the following Hölder estimate.

Lemma 2.9 (pointwise decay estimate for $\nabla G_{\eta}$ ). Let $a \in \mathcal{A}_{\alpha \beta}$, and $G_{\eta}$ be the associated Green's function. For all $d>2$, there exist $0<\gamma \leq 1$ and $c>0$ depending on $\alpha, \beta$, such that for all $x, y \in \mathbb{Z}^{d}$,

$$
\left|\nabla_{x} G_{\eta}(x, y)\right|,\left|\nabla_{y} G_{\eta}(x, y)\right| \lesssim \frac{1}{1+|x-y|^{d-2+\gamma}} \exp (-c|x-y| \sqrt{\eta}) .
$$


For $d=2$, there is a logarithmic correction:

$\left|\nabla_{x} G_{\eta}(x, y)\right|,\left|\nabla_{y} G_{\eta}(x, y)\right| \lesssim \min \left\{1, \frac{1}{1+|x-y|^{\gamma}}|\ln (\sqrt{\eta}(1+|x-y|))| \exp (-c|x-y| \sqrt{\eta})\right\}$.

Compared to Lemma 2.2, Lemma 2.7 does not distinguish between the diagonal case $(|x-z| \lesssim 1)$ and the off-diagonal case $(|x-z| \gg 1)$. This comes from the fact that the gradient of the Green's function is not singular in the discrete case.

Let us now turn to the weak norm of the fluctuation. The starting point is now

$$
\begin{array}{r}
\operatorname{var}\left[\int_{\varepsilon \mathbb{Z}^{d}} g(x) u_{\varepsilon}(x) d x\right]=\varepsilon^{4} \varepsilon^{2 d} \int_{\mathbb{Z}^{d}} \int_{\mathbb{Z}^{d}} \int_{\mathbb{Z}^{d}} \int_{\mathbb{Z}^{d}} g_{\varepsilon}(x) g_{\varepsilon}\left(x^{\prime}\right) f_{\varepsilon}(y) f_{\varepsilon}\left(y^{\prime}\right) \\
\operatorname{cov}\left[G_{\varepsilon^{2}}(x, y) ; G_{\varepsilon^{2}}\left(x^{\prime}, y^{\prime}\right)\right] d y d y^{\prime} d x d x^{\prime},
\end{array}
$$

where we have set $f_{\varepsilon}(x):=f(\varepsilon x)$ and $g_{\varepsilon}(x):=g(\varepsilon x)$ for all $x \in \mathbb{Z}^{d}$. In view of this quantity and of Lemma 2.7, it is not suprising that we shall need to estimate convolutions of the gradient of the Green's function with itself. As shown below, the estimates remain optimal (with respect to the Green's function of the Laplace operator):

Lemma 2.10 (convolution estimates). [7, Lemma 2.10] Let $h_{\eta}: \mathbb{Z}^{d} \rightarrow \mathbb{R}$ be such that for all $R \gg 1$ and $\eta>0$,

$$
\begin{array}{ll}
\text { for } d=2: & \int_{R<|z| \leq 2 R} h_{\eta}^{2}(z) d z \lesssim \min \left\{1,(\sqrt{\eta} R)^{-1}\right\}^{2}, \\
\text { for } d>2: & \int_{R<|z| \leq 2 R} h_{\eta}^{2}(z) d z \lesssim R^{2-d},
\end{array}
$$

and for $R \sim 1$

$$
\text { for } d \geq 2: \quad \int_{|z| \leq R} h_{\eta}^{2}(z) d z \lesssim 1
$$

Then for $R \gg 1$

$$
\begin{array}{ll}
\text { for } d=2: & \int_{|x| \leq R} \int_{\mathbb{Z}^{d}} h_{\eta}(z) h_{\eta}(z-x) d z d x \lesssim R^{2} \max \{1,-\ln (\sqrt{\eta} R)\}, \\
\text { for } d>2: & \int_{|x| \leq R} \int_{\mathbb{Z}^{d}} h_{\eta}(z) h_{\eta}(z-x) d z d x \lesssim R^{2} .
\end{array}
$$

The proof of Theorem 2 is much more technical than the proof of Theorem 1 . Theorem 3 can be proved using the same chain of arguments. However, the auxiliary lemmas need to be modified. These adaptations can be found in [6], and we do not reproduce them here. The only result we need here and didn't prove in [6] is the corresponding covariance estimate. We provide a statement and proof of the covariance estimate in the appendix.

\section{Proofs of Theorem 1 and of some auxiliary lemmas}

3.1. Proof of Theorem 1. We divide the proof into two steps, begin with the strong norm of the variance in the first step, and turn to the weak norm in the second step.

Step 1. Proof of (2.2). 
We recall that the change of variable $x \leadsto x / \varepsilon$ yields

$$
\int_{D} \operatorname{var}\left[u_{\varepsilon}(x)\right] d x=\varepsilon^{d} \int_{D / \varepsilon} \operatorname{var}\left[v_{\varepsilon}(x)\right] d x
$$

where $v_{\varepsilon}$ is the weak solution in $H_{0}^{1}(D / \varepsilon)$ to

$$
-\nabla \cdot\left(A_{\varepsilon}(x)+B(x)\right) \nabla v_{\varepsilon}(x)=\varepsilon^{2} f_{\varepsilon}(x)
$$

with

$$
\begin{aligned}
A_{\varepsilon}(x) & =A(\varepsilon x), \\
f_{\varepsilon}(x) & =f(\varepsilon x)
\end{aligned}
$$

and is given by $v_{\varepsilon}(x)=u_{\varepsilon}(\varepsilon x)$ for all $x \in D / \varepsilon$. Combined with the fact that $v_{\varepsilon}$ only depends on the restriction of $B$ on $D / \varepsilon$, Lemma 2.1 yields our starting point (2.14):

$$
\operatorname{var}\left[v_{\varepsilon}(x)\right] \lesssim \int_{\mathbb{R}^{d}}\left\langle\operatorname{Osc}_{\mid Q(z)}^{2} v_{\varepsilon}(x)\right\rangle d z
$$

We then appeal to the susceptibility estimate of Lemma 2.2 , and recall that we extend $f_{\varepsilon}$, $v_{\varepsilon}$ and $\nabla v_{\varepsilon}$, and $G$ and $\nabla G$ by zero on $\mathbb{R}^{d} \backslash D / \varepsilon$, and $\mathbb{R}^{d} \times \mathbb{R}^{d} \backslash(D / \varepsilon \times D / \varepsilon)$, respectively. This turns (2.14) into

$$
\begin{aligned}
\operatorname{var}\left[v_{\varepsilon}(x)\right] \lesssim \int_{|z-x| \geq 1}\left\langle\int_{Q(z)}\left|\nabla_{y} G(x, y)\right|^{2} d y\right. & \left.\int_{Q(z)}\left|\nabla v_{\varepsilon}(y)\right|^{2} d y\right\rangle d z \\
& +\int_{|z-x|<1} \int_{Q_{4}(z)}\left(\left|\nabla v_{\varepsilon}(y)\right|^{2}+\varepsilon^{4} f_{\varepsilon}(y)^{2}\right) d y d z .
\end{aligned}
$$

Using the pointwise bound (2.19) on $\nabla G$ in Lemma 2.4, and integrating on $x \in \mathbb{R}^{d}$, we may rewrite this inequality as

$$
\int_{\mathbb{R}^{d}} \operatorname{var}\left[v_{\varepsilon}(x)\right] \lesssim \int_{\mathbb{R}^{d}} \int_{\mathbb{R}^{d}} h_{\gamma}^{2}(z-x)\left\langle\int_{Q(z)}\left|\nabla v_{\varepsilon}(y)\right|^{2} d y\right\rangle d z d x+\varepsilon^{4} \int_{\mathbb{R}^{d}} f_{\varepsilon}(x)^{2} d x
$$

with

$$
h_{\gamma}(x)=\left\{\begin{array}{ccl}
1 & \text { if } & x \in 2 D / \varepsilon,|x|<1 \\
\frac{1}{|x|^{d-2+\gamma}} & \text { if } & x \in 2 D / \varepsilon,|x| \geq 1 \\
0 & \text { if } & x \notin 2 D / \varepsilon
\end{array}\right.
$$

Using Fubini's theorem, this yields

$$
\begin{aligned}
\int_{\mathbb{R}^{d}} \operatorname{var}\left[v_{\varepsilon}(x)\right] d x & \lesssim \int_{\mathbb{R}^{d}} h_{\gamma}^{2}(x) d x \int_{\mathbb{R}^{d}}\left\langle\int_{Q(z)}\left|\nabla v_{\varepsilon}(y)\right|^{2} d y\right\rangle d z+\varepsilon^{4} \int_{\mathbb{R}^{d}} f_{\varepsilon}(x)^{2} d x \\
& =\int_{\mathbb{R}^{d}} h_{\gamma}^{2}(x) d x\left\langle\int_{\mathbb{R}^{d}}\left|\nabla v_{\varepsilon}(z)\right|^{2} d z\right\rangle+\varepsilon^{4} \int_{\mathbb{R}^{d}} f_{\varepsilon}(x)^{2} d x
\end{aligned}
$$

By definition, the first factor of the first term of the r. h. s. is estimated by

$$
\int_{\mathbb{R}^{d}} h_{\gamma}^{2}(x) d x \lesssim\left\{\begin{array}{lll}
d=2 & : & \varepsilon^{-2+2 \gamma} \\
d=3 & : & \varepsilon^{\min \{0,2 \gamma-1\}} \\
d>3 & : & 1
\end{array}\right.
$$


For the second factor we appeal to equation (3.2) in the form of the a priori estimate

$$
\int_{D / \varepsilon}\left|\nabla v_{\varepsilon}(x)\right|^{2} d x \lesssim \varepsilon^{2} \int_{D / \varepsilon} f_{\varepsilon}(x) v_{\varepsilon}(x) d x .
$$

We perform the change of variables $x \leadsto \varepsilon x$, so that it turns into

$$
\int_{D / \varepsilon} f_{\varepsilon}(x) v_{\varepsilon}(x) d x=\varepsilon^{-d} \int_{D} f(x) u_{\varepsilon}(x) d x \lesssim \varepsilon^{-d}\|f\|_{L^{2}(D)}\left\|u_{\varepsilon}\right\|_{L^{2}(D)} .
$$

Using (2.1) and Poincaré's inequality on $D$, we then obtain

$$
\int_{D / \varepsilon}\left|\nabla v_{\varepsilon}(x)\right|^{2} d x \lesssim \varepsilon^{2-d}\|f\|_{L^{2}(D)}^{2}
$$

Likewise, the second term of the r. h. s. of (3.3) is controlled by

$$
\int_{D / \varepsilon} f_{\varepsilon}(x)^{2} d x \lesssim \varepsilon^{-d}\|f\|_{L^{2}(D)}^{2} .
$$

Hence,

$$
\int_{\mathbb{R}^{d}} \operatorname{var}\left[v_{\varepsilon}(x)\right] d x \lesssim\left\{\begin{array}{lll}
d=2 & : & \varepsilon^{-2+2 \gamma} \\
d=3 & : & \varepsilon^{2-d+\min \{0,2 \gamma-1\}}, \\
d>3 & : & \varepsilon^{2-d},
\end{array}\right.
$$

which, combined with (3.1), implies (2.2).

Step 2. Proof of (2.3).

Let $g \in L^{\infty}(D)$. The starting point is estimate $(2.20)$ :

$$
\operatorname{var}\left[\int_{D} u_{\varepsilon}(x) g(x) d x\right] \lesssim \varepsilon^{2 d} \int_{\mathbb{R}^{d}}\left\langle\left[\int_{D / \varepsilon}\left(\begin{array}{c}
\operatorname{Osc} \\
B_{\mid Q(z)}
\end{array} v_{\varepsilon}(x)\right)\left|g_{\varepsilon}(x)\right| d x\right]^{2}\right\rangle d z .
$$

Recall that $v_{\varepsilon}, f_{\varepsilon}$, and $g_{\varepsilon}$ are extended by 0 on $\mathbb{R}^{d} \backslash D / \varepsilon$. By the oscillation estimates of Lemma 2.2 and by Lemma 2.4,

$$
\begin{aligned}
\int_{\mathbb{R}^{d}}\left(\begin{array}{cc}
\operatorname{Osc} & \left.v_{\varepsilon}(x)\right)\left|g_{\varepsilon}(x)\right| d x \\
B_{\mid Q(z)}
\end{array}\right. \\
\quad \lesssim \int_{\mathbb{R}^{d}}\left[\left(\int_{Q_{4}(z)}\left|\nabla v_{\varepsilon}(y)\right|^{2} d y\right)^{1 / 2}+\left(\varepsilon^{4} \int_{Q_{4}(z)} f(y)^{2} d y\right)^{1 / 2}\right] h_{\gamma}(z-x)\left|g_{\varepsilon}(x)\right| d x,
\end{aligned}
$$

where

$$
h_{\gamma}(x)=\left\{\begin{array}{ccl}
1 & \text { if } & x \in 2 D / \varepsilon,|x|<1 \\
\frac{1}{|x|^{d-2+\gamma}} & \text { if } & x \in 2 D / \varepsilon,|x| \geq 1 \\
0 & \text { if } & x \notin 2 D / \varepsilon
\end{array}\right.
$$

Hence, expanding the square and using Young's inequality yield

$$
\begin{aligned}
\operatorname{var}\left[\int_{D} u_{\varepsilon}(x) g(x) d x\right] \lesssim \varepsilon^{2 d} \int_{\mathbb{R}^{d}} \int_{\mathbb{R}^{d}} \int_{\mathbb{R}^{d}} & \left\langle\int_{Q_{4}(z)}\left|\nabla v_{\varepsilon}(y)\right|^{2} d y+\varepsilon^{4} \int_{Q_{4}(z)} f(y)^{2} d y\right\rangle \\
& \times h_{\gamma}(z-x) h_{\gamma}\left(z-x^{\prime}\right)\left|g_{\varepsilon}(x)\right|\left|g_{\varepsilon}\left(x^{\prime}\right)\right| d x d x^{\prime} d z,
\end{aligned}
$$


By Fubini's theorem, this turns into

$\operatorname{var}\left[\int_{D} u_{\varepsilon}(x) g(x) d x\right] \lesssim \varepsilon^{2 d}\|g\|_{L^{\infty}}^{2}\left(\int_{\mathbb{R}^{d}} h_{\gamma}(x) d x\right)^{2}\left\langle\int_{\mathbb{R}^{d}}\left|\nabla v_{\varepsilon}(z)\right|^{2} d z+\varepsilon^{4} \int_{\mathbb{R}^{d}} f_{\varepsilon}(z)^{2} d z\right\rangle$.

Using then the a priori estimate (3.4), (3.5), and the definition of $h_{\gamma}$, we end up with

$$
\operatorname{var}\left[\int_{D} u_{\varepsilon}(x) g(x) d x\right] \lesssim \varepsilon^{2 d}\|g\|_{L^{\infty}}^{2}\left(\varepsilon^{-2+\gamma}\right)^{2} \varepsilon^{2-d}\|f\|_{L^{2}(D)}^{2} .
$$

This yields (2.3) and concludes the proof of the theorem.

3.2. Proof of Lemma 2.4. For $d>2$, Lemma 2.4 is a consequence of [9, (1.9) Theorem]. Since we did not find a suitable reference for $d=2$, we give the general argument, which is a simple combination of the pointwise estimates of Definition 2.6, Cacciopoli's inequality, and the deep Hölder regularity result of the De Giorgi-Nash-Moser theory in the form of [4, Theorem 8.27].

Let $x \in D / \varepsilon$. On the one hand, by the De Giorgi-Nash-Moser Hölder continuity result, for all $z \in D / \varepsilon$ with $|x-z|_{\infty}>1$, since $y \mapsto G(y, z)$ satisfies

$$
-\nabla \cdot A(y) \nabla_{y} G(y, z)=0
$$

on $Q_{|z-x| / 2}(x) \cap D / \varepsilon$ and $G(\cdot, z)=0$ on $\partial D / \varepsilon$, there exists $0<\gamma<1$ depending only on $\alpha, \beta$, and $d$ such that

$$
y \in Q_{2}(x) \cap D / \varepsilon=\underset{\text { osc }}{(x)} \cap(y, z) \lesssim|z-x|^{-\gamma} \sup _{Q_{|z-x| / 2}(x) \cap D / \varepsilon} G(\cdot, z)
$$

(see [4, Theorem 8.27] with " $\nu=0$ " and " $\sigma=0$ "). Combined with the pointwise decay estimates on $G$ from Definition 2.6 this yields

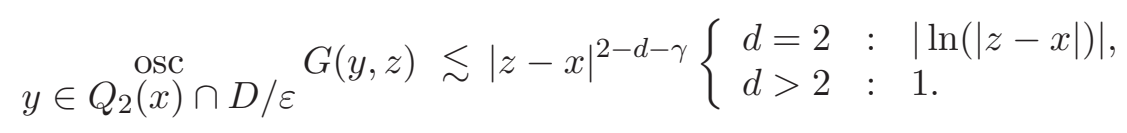

On the other hand, we appeal to Cacciopoli's inequality. We let $\eta: \mathbb{R}^{d} \rightarrow[0,1]$ be a smooth function such that $\eta(y)=1$ for all $y \in Q(x), \eta=0$ for all $y \in \mathbb{R}^{d} \backslash Q_{2}(x)$, and such that $|\nabla \eta| \lesssim 1$. We need to distinguish between points close to the boundary and points far from the boundary. We first treat the former, and consider $x \in D / \varepsilon$ such that $d_{\infty}(x, \partial D / \varepsilon)<1$ (where $d_{\infty}$ denotes the supremum distance in $\mathbb{R}^{d}$ ). We test the defining equation for $G(\cdot, z)$ with test-function $y \mapsto \eta^{2}(y) G(y, z)$, which belongs to $H_{0}^{1}(D / \varepsilon)$ since $G(\cdot, y)$ vanishes on $\partial D / \varepsilon$ and $\eta$ vanishes on a neighborhood of the singularity $y=z)$. This yields the Cacciopoli's estimate

$$
\int_{Q(x) \cap D / \cap D / \varepsilon}\left|\nabla_{y} G(y, z)\right|^{2} d y \lesssim \int_{Q_{2}(x) \cap D / \varepsilon} G(y, z)^{2} .
$$

Noting that $Q_{2}(x) \cap D / \varepsilon \neq \varnothing$, that $G(\cdot, z)>0$ on $D / \varepsilon$, and that $G(\cdot, z)=0$ on $\partial D / \varepsilon$, we have $\inf _{Q_{2}(x) \cap D / \varepsilon} G(\cdot, z)=0$ so that this estimate turns into

$$
\int_{Q(x) \cap D / \varepsilon}\left|\nabla_{y} G(y, z)\right|^{2} d y \lesssim\left(\underset{y \in Q_{2}(x) \cap D / \varepsilon}{\operatorname{osc}} G(y, z)\right)^{2} .
$$

The desired estimate (2.19) follows from (3.6) and (3.7) for $x \in D / \varepsilon$ such that $d_{\infty}(x, \partial D / \varepsilon)<$ 1. 
For points far from the boundary, namely for $x \in D / \varepsilon$ such that $d_{\infty}(x, \partial D / \varepsilon) \geq 1$ we have $Q_{2}(x) \subset D / \varepsilon$ so that $\eta$ is supported in $D / \varepsilon$. We then test the defining equation for $G(\cdot, z)$ with test-function $y \mapsto \eta^{2}(y)(G(y, z)-K) \in H_{0}^{1}(D / \varepsilon)$ for some $K \in \mathbb{R}$ to be fixed later (note that this function vanishes on a neighborhood of the singularity $y=z$ ). This yields the Cacciopoli's estimate

$$
\int_{Q(x)}\left|\nabla_{y} G(y, z)\right|^{2} d y \lesssim \int_{Q_{2}(x)}(G(y, z)-K)^{2} .
$$

Choosing $K=\inf _{Q_{2}(x)} G(\cdot, z)$, this turns into

$$
\int_{Q(x)}\left|\nabla_{y} G(y, z)\right|^{2} d y \lesssim\left(\underset{y \in Q_{2}(x)}{\operatorname{Osc}} G(y, z)\right)^{2} .
$$

The desired estimate (2.19) follows from (3.6) and (3.8) for $x \in D / \varepsilon$ such that $d_{\infty}(x, \partial D / \varepsilon) \geq$ 1.

3.3. Proof of Lemma 2.2. We split the proof into two steps and treat the cases $|z-x|>1$ and $|z-x| \leq 1$ separately. For notational convenience we replace the oscillation with respect to $B$ by the oscillation with respect to $C$. Let $z \in D / \varepsilon$, and $C$ and $\tilde{C}$ be two conductivity functions on $D / \varepsilon$ which coincide on $D / \varepsilon \backslash Q(z)$. We denote by $v_{\varepsilon}, \tilde{v}_{\varepsilon} \in$ $H_{0}^{1}(D / \varepsilon)$ the solutions to $(2.9)$ with conductivity matrices $C$ and $\tilde{C}$, respectively.

Step 1. Proof of (2.17)

By definition $\tilde{v}_{\varepsilon}-v_{\varepsilon}$ satisfies the equation

$$
-\nabla \cdot \tilde{C} \nabla\left(\tilde{v}_{\varepsilon}-v_{\varepsilon}\right)=\nabla \cdot(\tilde{C}-C) \nabla v_{\varepsilon} .
$$

Provided that $f$ and $C$ are smooth, $\nabla v_{\varepsilon} \in L^{\infty}(D / \varepsilon)$ and the function $\tilde{v}_{\varepsilon}-v_{\varepsilon}$ satisfies the Green representation formula

$$
\left(\tilde{v}_{\varepsilon}-v_{\varepsilon}\right)(x)=\int_{D / \varepsilon} \nabla \tilde{G}(x, y) \cdot(\tilde{C}(y)-C(y)) \nabla v_{\varepsilon}(y) d y,
$$

where $\tilde{G}$ denotes the Green's function associated with $\tilde{C}$ on $D / \varepsilon$ (with homogeneous Dirichlet boundary conditions). Since $\tilde{C}$ and $C$ coincide on $D / \varepsilon \backslash Q(z)$ this yields for all $x \in D / \varepsilon$ with $|x-z|>1$

$$
\left|\tilde{v}_{\varepsilon}(x)-v_{\varepsilon}(x)\right| \lesssim\left(\int_{Q(z)}|\nabla \tilde{G}(x, y)|^{2} d y\right)^{1 / 2}\left(\int_{Q(z)}\left|\nabla v_{\varepsilon}(y)\right|^{2} d y\right)^{1 / 2} .
$$

We need to take the supremum in $\left.C\right|_{Q(z)}$ and $\left.\tilde{C}\right|_{Q(z)}$. By [6, Lemma 2.9], we have for $|x-z| \geq 1$

$$
\sup _{\left.\tilde{C}\right|_{Q(z)}} \int_{Q(z)}|\nabla \tilde{G}(x, y)|^{2} d y \lesssim \int_{Q(z)}|\nabla G(x, y)|^{2} d y
$$

It remains to treat the second factor of the r. h. s. of (3.10). To this aim, we note that (3.9) yields the a priori estimate

$$
\int_{D / \varepsilon}\left|\nabla \tilde{v}_{\varepsilon}(y)-\nabla v_{\varepsilon}(y)\right|^{2} d y \lesssim \int_{Q(z)}\left|\nabla v_{\varepsilon}(y)\right|^{2} d y
$$


Hence, by the triangle inequality

$$
\left(\int_{Q(z)}\left|\nabla \tilde{v}_{\varepsilon}(y)\right|^{2} d y\right)^{1 / 2} \lesssim\left(\int_{Q(z)}\left|\nabla v_{\varepsilon}(y)\right|^{2} d y\right)^{1 / 2},
$$

so that

$$
\sup _{\left.C\right|_{Q(z)}} \int_{Q(z)}\left|\nabla v_{\varepsilon}(y)\right|^{2} d y \lesssim \int_{Q(z)}\left|\nabla v_{\varepsilon}(y)\right|^{2} d y
$$

The combination of (3.10), (3.11), and (3.12) proves the desired estimate (2.17) for $C$ and $f$ smooth. We conclude by regularization using Lemma 2.3 for general conductivity function $C$ and r. h. s. $f$.

Step 2. Proof of (2.18).

We consider $x, z \in D / \varepsilon$ such that $|x-z| \leq 1$, which corresponds to a diagonal estimate. To this aim we introduce two additional functions: $v_{\varepsilon}^{0}, \tilde{v}_{\varepsilon}^{0} \in H_{0}^{1}(D / \varepsilon)$ defined on $Q_{4}(z) \cap D / \varepsilon$ as the unique weak solutions in $H_{0}^{1}\left(Q_{4}(z) \cap D / \varepsilon\right)$ to

$$
\begin{aligned}
& -\nabla \cdot C \nabla v_{\varepsilon}^{0}=\varepsilon^{2} f_{\varepsilon}, \\
& -\nabla \cdot \tilde{C} \nabla \tilde{v}_{\varepsilon}^{0}=\varepsilon^{2} f_{\varepsilon},
\end{aligned}
$$

and extended by zero to $D / \varepsilon \backslash Q_{4}(z)$. We then split $v_{\varepsilon}^{0}$ into two parts $v_{\varepsilon}^{0,1}+v_{\varepsilon}^{0,2}$, defined on $Q_{4}(z) \cap D / \varepsilon$ as the unique weak solutions in $H_{0}^{1}\left(Q_{4}(z) \cap D / \varepsilon\right)$ to

$$
\begin{aligned}
& -\nabla \cdot \tilde{C} \nabla v_{\varepsilon}^{0,1}=\varepsilon^{2} f_{\varepsilon}-\nabla \cdot(\tilde{C}-C) \nabla v_{\varepsilon}, \\
& -\nabla \cdot \tilde{C} \nabla v_{\varepsilon}^{0,2}=-\nabla \cdot(\tilde{C}-C) \nabla\left(v_{\varepsilon}^{0}-v_{\varepsilon}\right),
\end{aligned}
$$

and extended by zero to $D / \varepsilon \backslash Q_{4}(z)$. We finally set

$$
\begin{aligned}
& \psi_{1}=\left(v_{\varepsilon}-v_{\varepsilon}^{0,1}\right)-\left(\tilde{v}_{\varepsilon}-\tilde{v}_{\varepsilon}^{0}\right), \\
& \psi_{2}=v_{\varepsilon}^{0}-\tilde{v}_{\varepsilon}^{0}, \\
& \psi_{3}=-v_{\varepsilon}^{0,2},
\end{aligned}
$$

so that

$$
v_{\varepsilon}-\tilde{v}_{\varepsilon}=\psi_{1}+\psi_{2}+\psi_{3}
$$

We treat each term separately.

We begin with the first term, and denote by $\tilde{G}$ and $\tilde{G}^{0}$ the Green's functions associated with $\tilde{C}$ and homogeneous Dirichlet boundary conditions on $D / \varepsilon$ and $Q_{4}(z) \cap D / \varepsilon$, respectively. Assuming that $C$ and $f$ are smooth so that $\nabla v_{\varepsilon} \in L^{\infty}(D / \varepsilon)$, from the equations

$$
\begin{array}{rlrl}
-\nabla \cdot \tilde{C} \nabla v_{\varepsilon} & =\varepsilon^{2} f_{\varepsilon}-\nabla \cdot(\tilde{C}-C) \nabla v_{\varepsilon}, & & \text { in } D / \varepsilon, \\
-\nabla \cdot \tilde{C} \nabla v_{\varepsilon}^{0,1} & =\varepsilon^{2} f_{\varepsilon}-\nabla \cdot(\tilde{C}-C) \nabla v_{\varepsilon}, & & \text { in } Q_{4}(z) \cap D / \varepsilon, \\
-\nabla \cdot \tilde{C} \nabla \tilde{v}_{\varepsilon} & =\varepsilon^{2} f_{\varepsilon}, \quad \text { in } D / \varepsilon, & \\
-\nabla \cdot \tilde{C} \nabla \tilde{v}_{\varepsilon}^{0} & =\varepsilon^{2} f_{\varepsilon}, \quad \text { in } Q_{4}(z) \cap D / \varepsilon, &
\end{array}
$$

we learn that $\psi_{1}$ satisfies the Green representation formula

$$
\psi_{1}(x)=\int_{Q_{4}(x) \cap D / \varepsilon} \nabla_{y}\left(\tilde{G}(x, y)-\tilde{G}^{0}(x, y)\right) \cdot(\tilde{C}(y)-C(y)) \nabla v_{\varepsilon}(y) d y .
$$


Hence, since $\tilde{C}=C$ on $D / \varepsilon \backslash Q(z)$,

$$
\left|\psi_{1}(x)\right| \lesssim\left(\int_{Q(x) \cap D / \varepsilon}\left|\nabla_{y}\left(\tilde{G}(x, y)-\tilde{G}^{0}(x, y)\right)\right|^{2} d y\right)^{1 / 2}\left(\int_{Q(x) \cap D / \varepsilon}\left|\nabla v_{\varepsilon}(y)\right|^{2} d y\right)^{1 / 2} .
$$

We need to take the supremum in $\left.\tilde{C}\right|_{Q(z)}$ and $\left.C\right|_{Q(z)}$. For the second factor of the r. h. s. we appeal to $(3.12)$ in Step 1. For the first factor, we use that $\tilde{G}(\cdot, x)-\tilde{G}^{0}(\cdot, x)$ satisfies the equation

$$
-\nabla_{y} \cdot \tilde{C}(y) \nabla_{y}\left(\tilde{G}(y, x)-\tilde{G}^{0}(y, x)\right)=0 \quad \text { in } Q_{4}(z) \cap D / \varepsilon,
$$

so that Cacciopoli's inequality yields

$$
\int_{Q(z) \cap D / \varepsilon}\left|\nabla_{y}\left(\tilde{G}(y, x)-\tilde{G}^{0}(y, x)\right)\right|^{2} d y \lesssim \int_{Q_{2}(z)}\left(\tilde{G}(y, x)-\tilde{G}^{0}(y, x)\right)^{2} d y,
$$

whereas the maximum principle yields

$$
\int_{Q_{4}(z) \cap D / \varepsilon}\left(\tilde{G}(y, x)-\tilde{G}^{0}(y, x)\right)^{2} d y \lesssim \sup _{\partial\left(Q_{4}(z) \cap D / \varepsilon\right)} G(\cdot, x)^{2} .
$$

Since $|x-z| \leq 1$, the combination of (3.14) and (3.15) with the pointwise decay (2.16) in Definition 2.6 and the symmetry of $\tilde{G}$ and $\tilde{G}^{0}$ shows that

$$
\int_{Q(z) \cap D / \varepsilon}\left|\nabla_{y}\left(\tilde{G}(x, y)-\tilde{G}^{0}(x, y)\right)\right|^{2} d y \lesssim 1,
$$

from which we conclude that for $C$ and $f$ smooth,

$$
\sup _{\left.C\right|_{Q(z)},\left.\tilde{C}\right|_{Q(z)}}\left|\psi_{1}(x)\right| \lesssim\left(\int_{Q(x) \cap D / \varepsilon}\left|\nabla v_{\varepsilon}(y)\right|^{2} d y\right)^{1 / 2} .
$$

We turn to the second term $\psi_{2}$. Provided $f$ is smooth, for all $x \in Q_{4}(z), \psi_{2}$ satisfies the Green representation formula

$$
\psi_{2}(x)=\varepsilon^{2} \int_{Q_{4}(z)}\left(G^{0}(x, y)-\tilde{G}^{0}(x, y)\right) f_{\varepsilon}(y) d y .
$$

Combined with Cauchy-Schwarz' inequality, the maximum principle in the form of (3.15) then yields

$$
\left|\psi_{2}(x)\right| \lesssim \varepsilon^{2}\left(\int_{Q_{4}(z)} f_{\varepsilon}(y)^{2} d y\right)^{1 / 2}
$$

whose r. h. s. is independent of $C$ and $\tilde{C}$.

We turn to the third and last term $\psi_{3}$. We rewrite the equation for $\psi_{3}$ as

$$
-\nabla \cdot \tilde{C} \nabla\left(\psi_{3}-v_{\varepsilon}+v_{\varepsilon}^{0}+f_{Q_{4}(z) \cap D / \varepsilon} v_{\varepsilon}\right)=0 \quad \text { in } Q_{4}(z) \cap D / \varepsilon .
$$


From [4, Theorem 8.25] we have the boundedness estimate

$$
\begin{aligned}
\sup _{Q_{2}(z) \cap D / \varepsilon}\left|\psi_{3}-v_{\varepsilon}+v_{\varepsilon}^{0}+f_{Q_{4}(z) \cap D / \varepsilon} v_{\varepsilon}\right| \\
\quad \lesssim\left\|\psi_{3}\right\|_{L^{2}\left(Q_{4}(z) \cap D / \varepsilon\right)}+\left\|v_{\varepsilon}^{0}\right\|_{L^{2}\left(Q_{4}(z) \cap D / \varepsilon\right)}+\left\|v_{\varepsilon}-f_{Q_{4}(z) \cap D / \varepsilon} v_{\varepsilon}\right\|_{L^{2}\left(Q_{4}(z) \cap D / \varepsilon\right)} .
\end{aligned}
$$

By Poincaré's inequality, this turns into

$$
\begin{aligned}
\sup _{Q_{2}(z) \cap D / \varepsilon} \mid \psi_{3}-v_{\varepsilon} & +v_{\varepsilon}^{0}+f_{Q_{4}(z) \cap D / \varepsilon} v_{\varepsilon} \mid \\
& \lesssim\left\|\nabla \psi_{3}\right\|_{L^{2}\left(Q_{4}(z) \cap D / \varepsilon\right)}+\left\|\nabla v_{\varepsilon}^{0}\right\|_{L^{2}\left(Q_{4}(z) \cap D / \varepsilon\right)}+\left\|\nabla v_{\varepsilon}\right\|_{L^{2}\left(Q_{4}(z) \cap D / \varepsilon\right)} .
\end{aligned}
$$

From the defining equation for $v_{\varepsilon}^{0,2}$, we have the a priori estimate

$$
\left\|\nabla \psi_{3}\right\|_{L^{2}\left(Q_{4}(z) \cap D / \varepsilon\right)} \lesssim\left\|\nabla v_{\varepsilon}^{0}\right\|_{L^{2}\left(Q_{4}(z) \cap D / \varepsilon\right)}+\left\|\nabla v_{\varepsilon}\right\|_{L^{2}\left(Q_{4}(z) \cap D / \varepsilon\right)} .
$$

Likewise, from the defining equation for $v_{\varepsilon}^{0}$ combined with Poincaré's inequality we deduce the a priori estimate

$$
\left\|\nabla v_{\varepsilon}^{0}\right\|_{L^{2}\left(Q_{4}(z) \cap D / \varepsilon\right)} \lesssim \varepsilon^{2}\left\|f_{\varepsilon}\right\|_{L^{2}\left(Q_{4}(z) \cap D / \varepsilon\right)} .
$$

Hence the boundedness estimate takes the form

$$
\begin{aligned}
\sup _{Q_{2}(z) \cap D / \varepsilon}\left|\psi_{3}\right| \lesssim & \sup _{Q_{2}(z) \cap D / \varepsilon}\left|v_{\varepsilon}-v_{\varepsilon}^{0}-f_{Q_{4}(z) \cap D / \varepsilon} v_{\varepsilon}\right| \\
& +\left(\int_{Q_{4}(z) \cap D / \varepsilon}\left|\nabla v_{\varepsilon}(y)\right|^{2} d y\right)^{1 / 2}+\varepsilon^{2}\left(\int_{Q_{4}(z) \cap D / \varepsilon} f_{\varepsilon}(y)^{2} d y\right)^{1 / 2} .
\end{aligned}
$$

Noting that

$$
-\nabla \cdot C \nabla\left(v_{\varepsilon}-v_{\varepsilon}^{0}-f_{Q_{4}(z) \cap D / \varepsilon} v_{\varepsilon}\right)=0 \quad \text { in } Q_{4}(z) \cap D / \varepsilon,
$$

we may proceed as above, appeal to [4, Theorem 8.25], and deduce that

$$
\begin{aligned}
\sup _{Q_{2}(z) \cap D / \varepsilon} \mid v_{\varepsilon}-v_{\varepsilon}^{0}- & f_{Q_{4}(z) \cap D / \varepsilon} v_{\varepsilon} \mid \\
& \lesssim\left(\int_{Q_{4}(z) \cap D / \varepsilon}\left|\nabla v_{\varepsilon}(y)\right|^{2} d y\right)^{1 / 2}+\varepsilon^{2}\left(\int_{Q_{4}(z) \cap D / \varepsilon} f_{\varepsilon}(y)^{2} d y\right)^{1 / 2},
\end{aligned}
$$

so that (3.18) turns into

$$
\sup _{Q_{2}(z) \cap D / \varepsilon}\left|\psi_{3}\right| \lesssim\left(\int_{Q_{4}(z) \cap D / \varepsilon}\left|\nabla v_{\varepsilon}(y)\right|^{2} d y\right)^{1 / 2}+\varepsilon^{2}\left(\int_{Q_{4}(z) \cap D / \varepsilon} f_{\varepsilon}(y)^{2} d y\right)^{1 / 2} .
$$


From the combination of (3.13) with (3.16), (3.17), and (3.19), we deduce that for $f$ smooth,

$$
\sup _{Q_{2}(z) \cap D / \varepsilon}\left|v_{\varepsilon}-\tilde{v}_{\varepsilon}\right| \lesssim\left(\int_{Q_{4}(z) \cap D / \varepsilon}\left|\nabla v_{\varepsilon}(y)\right|^{2} d y\right)^{1 / 2}+\varepsilon^{2}\left(\int_{Q_{4}(z) \cap D / \varepsilon} f_{\varepsilon}(y)^{2} d y\right)^{1 / 2} .
$$

The desired estimate (2.18) then follows for $f$ smooth from taking the supremum in $\left.C\right|_{Q_{4}(z) \cap D / \varepsilon}$ using (3.12), and extends to general $f$ using Lemma 2.3.

3.4. Proof of Lemma 2.3. The proof of Property (a) is the same as in [6, Lemma 2.7], and we only prove Property (b).

The function $v_{\varepsilon}^{h}-v_{\varepsilon} \in H_{0}^{1}(D / \varepsilon)$ is solution to

$$
-\nabla \cdot C_{h} \nabla\left(v_{\varepsilon}^{h}-v_{\varepsilon}\right)=\left(f_{\varepsilon}^{h}-f_{\varepsilon}\right)-\nabla \cdot\left(C_{h}-C\right) \nabla v_{\varepsilon} .
$$

Multiplying by $v_{\varepsilon}^{h}-v_{\varepsilon}$ and integrating by parts yield the a priori estimate

$$
\int_{D / \varepsilon}\left|\nabla\left(v_{\varepsilon}^{h}-v_{\varepsilon}\right)\right|^{2} \lesssim \int_{D / \varepsilon}\left|f_{\varepsilon}^{h}-f_{\varepsilon}\right|\left|v_{\varepsilon}^{h}-v_{\varepsilon}\right|+\int_{D / \varepsilon}\left|C_{h}-C\right|\left|\nabla v_{\varepsilon}\right|\left|\nabla\left(v_{\varepsilon}^{h}-v_{\varepsilon}\right)\right| .
$$

By Cauchy-Schwarz', Poincaré's and Young's inequalities, this turns into

$$
\int_{D / \varepsilon}\left|\nabla\left(v_{\varepsilon}^{h}-v_{\varepsilon}\right)\right|^{2} \lesssim \int_{D / \varepsilon}\left|f_{\varepsilon}^{h}-f_{\varepsilon}\right|^{2}+\int_{D / \varepsilon}\left|C_{h}-C\right|^{2}\left|\nabla v_{\varepsilon}\right|^{2} .
$$

We conclude by the dominated convergence theorem that

$$
\lim _{h \rightarrow 0} \int_{D / \varepsilon}\left|\nabla\left(v_{\varepsilon}^{h}-v_{\varepsilon}\right)\right|^{2}=0,
$$

so that by Poincaré's inequality, $v_{\varepsilon}^{h}$ converges to $v_{\varepsilon}$ in $H^{1}(D / \varepsilon)$. By the De Giorgi-NashMoser Hölder regularity theory, this implies that $v_{\varepsilon}^{h}$ converges to $v_{\varepsilon}$ pointwise everywhere on $D / \varepsilon$.

\section{Proof of Theorem 2 and of Lemma 2.9}

4.1. Proof of Theorem 2. We divide the proof into two steps, and first address the strong estimate of the fluctuation.

Step 1. Proof of (2.6).

The starting point is the Green representation formula

$$
u_{\varepsilon}(\varepsilon x)=\varepsilon^{2} \int_{\mathbb{Z}^{d}} G_{\varepsilon^{2}}(x, y) f(\varepsilon y) d y,
$$

from which we deduce

$$
\int_{\varepsilon \mathbb{Z}^{d}} \operatorname{var}\left[u_{\varepsilon}(x)\right] d x=\varepsilon^{d+4} \int_{\mathbb{Z}^{d}} \int_{\mathbb{Z}^{d}} \int_{\mathbb{Z}^{d}} f(\varepsilon y) f\left(\varepsilon y^{\prime}\right) \operatorname{cov}\left[G_{\varepsilon^{2}}(x, y) ; G_{\varepsilon^{2}}\left(x, y^{\prime}\right)\right] d y d y^{\prime} d x .
$$

From Lemmas 2.6 and 2.5, we learn that for all $x, y, y^{\prime} \in \mathbb{Z}^{d}$, the covariance in the integrand of (4.2) is estimated by

$$
\left.\operatorname{cov}\left[G_{\varepsilon^{2}}(x, y) ; G_{\varepsilon^{2}}\left(x, y^{\prime}\right)\right] \lesssim \sum_{e}\left\langle\sup _{b(e)}\left|\frac{\partial G_{\varepsilon^{2}}(x, y)}{\partial b(e)}\right|^{2}\right\rangle^{2}\right\rangle^{1 / 2}\left\langle\sup _{b(e)}\left|\frac{\partial G_{\varepsilon^{2}}\left(x, y^{\prime}\right)}{\partial b(e)}\right|^{2}\right\rangle^{1 / 2},
$$


where the sum runs over the edges of $\mathbb{Z}^{d}$. We then appeal to Lemma 2.7 to turn (4.3) into

$$
\begin{aligned}
\operatorname{cov}\left[G_{\varepsilon^{2}}(x, y) ; G_{\varepsilon^{2}}\left(x, y^{\prime}\right)\right] \lesssim \int_{\mathbb{Z}^{d}}\left\langle\left|\nabla_{z} G_{\varepsilon^{2}}(x, z)\right|^{2}\left|\nabla_{z} G_{\varepsilon^{2}}(z, y)\right|^{2}\right\rangle^{1 / 2} \\
\\
\quad \times\left\langle\left|\nabla_{z} G_{\varepsilon^{2}}(x, z)\right|^{2}\left|\nabla_{z} G_{\varepsilon^{2}}\left(z, y^{\prime}\right)\right|^{2}\right\rangle^{1 / 2} d z .
\end{aligned}
$$

Without loss of generality, we assume that the Meyers' exponent of Lemma 2.8 satisfies $2<p \leq 4$. We use Hölder's inequality in probability on both terms, with exponents $(p /(p-2), p / 2)$. This yields

$$
\begin{aligned}
\operatorname{cov}\left[G_{\varepsilon^{2}}(x, y) ; G_{\varepsilon^{2}}\left(x, y^{\prime}\right)\right] \lesssim \int_{\mathbb{Z}^{d}}\left\langle\left|\nabla_{z} G_{\varepsilon^{2}}(x, z)\right|^{2 p /(p-2)}\right\rangle^{(p-2) / p}\left\langle\left|\nabla_{z} G_{\varepsilon^{2}}(z, y)\right|^{p}\right\rangle^{1 / p} \\
\times\left\langle\left|\nabla_{z} G_{\varepsilon^{2}}\left(z, y^{\prime}\right)\right|^{p}\right\rangle^{1 / p} d z .
\end{aligned}
$$

We now insert (4.4) into (4.2), and obtain

$$
\begin{aligned}
\int_{\varepsilon \mathbb{Z}^{d}} \operatorname{var}\left[u_{\varepsilon}(x)\right] d x \lesssim \varepsilon^{d+4} \int_{\mathbb{Z}^{d}} \int_{\mathbb{Z}^{d}} \int_{\mathbb{Z}^{d}} \int_{\mathbb{Z}^{d}} f(\varepsilon y) f\left(\varepsilon y^{\prime}\right)\left\langle\left|\nabla_{z} G_{\varepsilon^{2}}(x, z)\right|^{2 p /(p-2)}\right\rangle^{(p-2) / p} \\
\\
\times\left\langle\left|\nabla_{z} G_{\varepsilon^{2}}(z, y)\right|^{p}\right\rangle^{1 / p}\left\langle\left|\nabla_{z} G_{\varepsilon^{2}}\left(z, y^{\prime}\right)\right|^{p}\right\rangle^{1 / p} d z d y d y^{\prime} d x .
\end{aligned}
$$

Using Fubini's theorem, we rewrite this inequality as

$$
\begin{aligned}
\int_{\varepsilon \mathbb{Z}^{d}} \operatorname{var}\left[u_{\varepsilon}(x)\right] d x \lesssim \varepsilon^{d+4} \int_{\mathbb{Z}^{d}} & \int_{\mathbb{Z}^{d}} \int_{\mathbb{Z}^{d}} f(\varepsilon y) f\left(\varepsilon y^{\prime}\right)\left\langle\left|\nabla_{z} G_{\varepsilon^{2}}(z, y)\right|^{p}\right\rangle^{1 / p}\left\langle\left|\nabla_{z} G_{\varepsilon^{2}}\left(z, y^{\prime}\right)\right|^{p}\right\rangle^{1 / p} \\
& \times\left(\int_{\mathbb{Z}^{d}}\left\langle\left|\nabla_{z} G_{\varepsilon^{2}}(x, z)\right|^{2 p /(p-2)}\right\rangle^{(p-2) / p} d x\right) d z d y d y^{\prime} . \quad \text { (4.5) }
\end{aligned}
$$

We first estimate the last term of the r. h. s. of (4.5). By stationarity,

$$
\left\langle\left|\nabla_{z} G_{\varepsilon^{2}}(x, z)\right|^{2 p /(p-2)}\right\rangle=\left\langle\left|\nabla_{z} G_{\varepsilon^{2}}(0, z-x)\right|^{2 p /(p-2)}\right\rangle .
$$

Denoting by $R \gg 1$ a radius such that the higher integrability of Lemma 2.8 holds, we decompose the integral over $\mathbb{Z}^{d}$ as the integral over the ball $\{|x| \leq R\}$ and the integrals over dyadic annuli $\left\{2^{i} R<|x| \leq 2^{i+1} R\right\}$ for $i \in \mathbb{N}$. For the integral over the ball $\{|x| \leq R\}$ we appeal to the pointwise estimate of Lemma 2.9 to get

$$
\int_{|x-z| \leq R}\left\langle\left|\nabla_{z} G_{\varepsilon^{2}}(0, z-x)\right|^{2 p /(p-2)}\right\rangle^{(p-2) / p} d x \lesssim 1
$$

For the integral over the dyadic annuli, we shall estimate a $p$-th power of the gradient of the Green's function using the (optimal) higher integrability of Lemma 2.8, and the remaining part by the (suboptimal) pointwise estimate of Lemma 2.9. In particular, from the elementary identity $2 p /(p-2)=p+p(4-p) /(p-2)$ and Lemma 2.9, we get for $d>2$

$$
\begin{aligned}
& \int_{2^{i} R<|x-z| \leq 2^{i+1} R}\left\langle\left|\nabla_{z} G_{\varepsilon^{2}}(x, z)\right|^{2 p /(p-2)}\right\rangle^{(p-2) / p} d x \\
& \quad=\int_{2^{i} R<|x-z| \leq 2^{i+1} R}\left\langle\left|\nabla_{z} G_{\varepsilon^{2}}(x, z)\right|^{p(4-p) /(p-2)}\left|\nabla_{z} G_{\varepsilon^{2}}(x, z)\right|^{p}\right\rangle^{(p-2) / p} d x \\
& \quad \lesssim \int_{2^{i} R<|x-z| \leq 2^{i+1} R}\left(\frac{1}{1+|x-z|^{d+\gamma-2}} \exp (-c \varepsilon|x-z|)\right)^{4-p}\left\langle\left|\nabla_{z} G_{\varepsilon^{2}}(x, z)\right|^{p}\right\rangle^{(p-2) / p} d x .
\end{aligned}
$$


Combined with Hölder's inequality with exponents $(p / 2, p /(p-2))$ and the estimate $(2.26)$ of Lemma 2.8 for $d>2$, this turns into

$$
\begin{aligned}
& \int_{2^{i} R<|x-z| \leq 2^{i+1} R}\left\langle\left|\nabla_{z} G_{\varepsilon^{2}}(x, z)\right|^{2 p /(p-2)}\right\rangle^{(p-2) / p} d x \\
& \quad \lesssim \quad\left(2^{i}\right)^{(2-d-\gamma)(4-p)}\left(2^{i}\right)^{2 d / p} \exp \left(-c \varepsilon 2^{i}\right)\left\langle\int_{2^{i} R \leq|x-z|<2^{i+1} R}\left|\nabla_{z} G_{\varepsilon^{2}}(x, z)\right|^{p} d x\right\rangle^{(p-2) / p} \\
& \quad \stackrel{(2.26)}{\lesssim}\left(2^{i}\right)^{(2-d-\gamma)(4-p)}\left(2^{i}\right)^{2 d / p} \exp \left(-c \varepsilon 2^{i}\right)\left(2^{i}\right)^{(d+(1-d) p)(p-2) / p} \\
& \quad=\left(2^{i}\right)^{2-d+(4-p)(1-\gamma)} \exp \left(-c \varepsilon 2^{i}\right) .
\end{aligned}
$$

For $d=2$, there is an additional logarithmic correction:

$$
\begin{gathered}
\int_{2^{i} R<|x-z| \leq 2^{i+1} R}\left\langle\left|\nabla_{z} G_{\varepsilon^{2}}(x, z)\right|^{2 p /(p-2)}\right\rangle^{(p-2) / p} d x \\
\lesssim \int_{2^{i} R<|x-z| \leq 2^{i+1} R}\left|\ln \left(\frac{\varepsilon^{-1}}{1+|x-z|}\right)\right|^{4-p} \\
\quad \times\left(\frac{1}{1+|x-z|^{\gamma}} \exp (-c \varepsilon|x-z|)\right)^{4-p}\left\langle\left|\nabla_{z} G_{\varepsilon^{2}}(x, z)\right|^{p}\right\rangle^{(p-2) / p} d x \\
\lesssim|\ln \varepsilon|^{4-p} \int_{2^{i} R \leq|x-z|<2^{i+1} R}\left(\frac{1}{1+|x-z|^{\gamma}} \exp (-c \varepsilon|x-z|)\right)^{4-p} \\
\times\left\langle\left|\nabla_{z} G_{\varepsilon^{2}}(x, z)\right|^{p}\right\rangle^{(p-2) / p} d x .
\end{gathered}
$$

For $d=2,(4.7)$ is thus replaced by

$$
\int_{2^{i} R<|x-z| \leq 2^{i+1} R}\left\langle\left|\nabla_{z} G_{\varepsilon^{2}}(x, z)\right|^{2 p /(p-2)}\right\rangle^{(p-2) / p} d x \lesssim|\ln \varepsilon|^{4-p}\left(2^{i}\right)^{(4-p)(1-\gamma)} \exp \left(-c \varepsilon 2^{i}\right) .
$$

The combination of (4.7), (4.8), and (4.6) with the dyadic decomposition of space then yields

$$
\begin{aligned}
& \int_{\mathbb{Z}^{d}}\left\langle\left|\nabla_{z} G_{\varepsilon^{2}}(x, z)\right|^{2 p /(p-2)}\right\rangle^{(p-2) / p} d x \\
& \lesssim\left\{\begin{array}{lll}
d=2 & : & \max \left\{|\ln \varepsilon|,\left(\varepsilon^{-(1-\gamma)}|\ln \varepsilon|\right)^{\max \{0,4-p\}}\right\}, \\
d=3: & : \begin{cases}\varepsilon^{p-3+\frac{\gamma}{1-\gamma}} & \text { for } p<3-\frac{\gamma}{1-\gamma}, \\
|\ln \varepsilon| & \text { for } p=3-\frac{\gamma}{1-\gamma}, \\
1 & \text { for } p>3-\frac{\gamma}{1-\gamma},\end{cases} \\
d>3: & 1 . &
\end{array}\right. \\
& 24
\end{aligned}
$$


We now turn to the estimate of the triple integral in (4.5). By stationarity and symmetry of $y$ and $y^{\prime}$,

$$
\begin{aligned}
& \int_{\mathbb{Z}^{d}} \int_{\mathbb{Z}^{d}} \int_{\mathbb{Z}^{d}} f(\varepsilon y) f\left(\varepsilon y^{\prime}\right)\left\langle\left|\nabla_{z} G_{\varepsilon^{2}}(z, y)\right|^{p}\right\rangle^{1 / p}\left\langle\left|\nabla_{z} G_{\varepsilon^{2}}\left(z, y^{\prime}\right)\right|^{p}\right\rangle^{1 / p} d z d y d y^{\prime} \\
& \quad \leq \int_{\mathbb{Z}^{d}} \int_{\mathbb{Z}^{d}} \int_{\mathbb{Z}^{d}} f(\varepsilon y)^{2}\left\langle\left|\nabla_{z} G_{\varepsilon^{2}}(z, y)\right|^{p}\right\rangle^{1 / p}\left\langle\left|\nabla_{z} G_{\varepsilon^{2}}\left(z, y^{\prime}\right)\right|^{p}\right\rangle^{1 / p} d z d y d y^{\prime} \\
& \quad=\int_{\mathbb{Z}^{d}} f(\varepsilon y)^{2} \int_{\mathbb{Z}^{d}} \int_{\mathbb{Z}^{d}}\left\langle\left|\nabla_{z} G_{\varepsilon^{2}}(z, 0)\right|^{p}\right\rangle^{1 / p}\left\langle\left|\nabla_{z} G_{\varepsilon^{2}}\left(z, y^{\prime}-y\right)\right|^{p}\right\rangle^{1 / p} d z d y^{\prime} d y \\
& \quad \lesssim \varepsilon^{-d}\|f\|_{2}^{2} \int_{\mathbb{Z}^{d}} \int_{\mathbb{Z}^{d}}\left\langle\left|\nabla_{z} G_{\varepsilon^{2}}(z, 0)\right|^{p}\right\rangle^{1 / p}\left\langle\left|\nabla_{z} G_{\varepsilon^{2}}\left(z, y^{\prime}\right)\right|^{p}\right\rangle^{1 / p} d z d y^{\prime}
\end{aligned}
$$

We then decompose the space into $\left\{\left|y^{\prime}\right| \geq \varepsilon^{-1}\right\}$ - for which we use the exponential decay - and $\left\{\left|y^{\prime}\right|<\varepsilon^{-1}\right\}$ - for which we appeal to the convolution estimate of Lemma 2.10. We begin with the first term. Either

$$
\left|y^{\prime}\right| \geq \varepsilon^{-1} \quad \text { and } \quad|z| \leq\left|z-y^{\prime}\right| \quad \Longrightarrow \quad\left|z-y^{\prime}\right| \geq \varepsilon^{-1} / 2 \quad \text { and } \quad z \in \mathbb{Z}^{d},
$$

or

$$
\left|y^{\prime}\right| \geq \varepsilon^{-1} \quad \text { and } \quad|z|>\left|z-y^{\prime}\right| \quad \Longrightarrow \quad|z| \geq \varepsilon^{-1 / 2} \quad \text { and } \quad z-y^{\prime} \in \mathbb{Z}^{d},
$$

so that by symmetry of the roles of $z$ and $z-y^{\prime}$ (which follows from the stationarity of the Green's function), we have

$$
\begin{aligned}
& \int_{\left|y^{\prime}\right| \geq \varepsilon^{-1}} \int_{\mathbb{Z}^{d}}\left\langle\left|\nabla_{z} G_{\varepsilon^{2}}(z, 0)\right|^{p}\right\rangle^{1 / p}\left\langle\left|\nabla_{z} G_{\varepsilon^{2}}\left(z, y^{\prime}\right)\right|^{p}\right\rangle^{1 / p} d z d y^{\prime} \\
& \quad \leq 2 \int_{\left|y^{\prime}\right| \geq \varepsilon^{-1}} \int_{|z| \leq\left|z-y^{\prime}\right|}\left\langle\left|\nabla_{z} G_{\varepsilon^{2}}(z, 0)\right|^{p}\right\rangle^{1 / p}\left\langle\left|\nabla_{z} G_{\varepsilon^{2}}\left(z, y^{\prime}\right)\right|^{p}\right\rangle^{1 / p} d z d y^{\prime} \\
& \quad \lesssim \int_{z \in \mathbb{Z}^{d}} \int_{\left|z-y^{\prime}\right| \geq \varepsilon^{-1} / 2}\left\langle\left|\nabla_{z} G_{\varepsilon^{2}}(z, 0)\right|^{p}\right\rangle^{1 / p}\left\langle\left|\nabla_{z} G_{\varepsilon^{2}}\left(z, y^{\prime}\right)\right|^{p}\right\rangle^{1 / p} d y^{\prime} d z .
\end{aligned}
$$

From the stationarity of $\nabla G_{\varepsilon^{2}}$ and a dydadic decomposition of space combined with the decay estimate of Lemma 2.8 and the uniform bound $\left|\nabla G_{\varepsilon^{2}}\right| \lesssim 1$ of Lemma 2.9, we learn that this integral behaves as if $\left\langle\left|\nabla_{z} G_{\varepsilon^{2}}(z, 0)\right|^{p}\right\rangle^{1 / p}$ decayed pointwise as $\frac{\exp (-c \varepsilon|z|)}{1+|z|^{\mid d-1}}$, so that

$$
\begin{aligned}
& \int_{\left|y^{\prime}\right| \geq \varepsilon^{-1}} \int_{\mathbb{Z}^{d}}\left\langle\left|\nabla_{z} G_{\varepsilon^{2}}(z, 0)\right|^{p}\right\rangle^{1 / p}\left\langle\left|\nabla_{z} G_{\varepsilon^{2}}\left(z, y^{\prime}\right)\right|^{p}\right\rangle^{1 / p} d z d y^{\prime} \\
& \quad \lesssim\left(\int_{z \in \mathbb{Z}^{d}} \frac{\exp (-c \varepsilon|z|)}{1+|z|^{d-1}} d z\right)\left(\int_{\left|z^{\prime}\right| \geq \varepsilon^{-1} / 2} \frac{\exp \left(-c \varepsilon\left|z^{\prime}\right|\right)}{1+\left|z^{\prime}\right|^{d-1}} d z^{\prime}\right) \\
& \quad \lesssim\left(\int_{t=0}^{\infty} \exp (-c \varepsilon t) d t\right)\left(\int_{t=\varepsilon^{-1}}^{\infty} \exp (-c \varepsilon t) d t\right) \\
& \quad \lesssim 1+\varepsilon^{-2}
\end{aligned}
$$

We now turn to the integral over $\left\{\left|y^{\prime}\right|<\varepsilon^{-1}\right\}$. Setting $h(z):=\left\langle\left|\nabla_{z} G_{\varepsilon^{2}}(z, 0)\right|^{p}\right\rangle^{1 / p}$ and using the stationarity of $\nabla G_{\varepsilon^{2}}$, we are in position to apply the convolution estimate of Lemma 2.10 with $\eta=\varepsilon^{2}$ and $R=\varepsilon^{-1}$, which yields

$$
\int_{\left|y^{\prime}\right|<\varepsilon^{-1}} \int_{\mathbb{Z}^{d}}\left\langle\left|\nabla_{z} G_{\varepsilon^{2}}(z, 0)\right|^{p}\right\rangle^{1 / p}\left\langle\left|\nabla_{z} G_{\varepsilon^{2}}\left(z, y^{\prime}\right)\right|^{p}\right\rangle^{1 / p} d z d y^{\prime} \lesssim 1+\varepsilon^{-2} .
$$


From (4.11) \& (4.12), we deduce

$$
\int_{\mathbb{Z}^{d}} \int_{\mathbb{Z}^{d}}\left\langle\left|\nabla_{z} G_{\varepsilon^{2}}(z, 0)\right|^{p}\right\rangle^{1 / p}\left\langle\left|\nabla_{z} G_{\varepsilon^{2}}\left(z, y^{\prime}\right)\right|^{p}\right\rangle^{1 / p} d z d y^{\prime} \lesssim 1+\varepsilon^{-2} .
$$

Combined with (4.5), (4.9), and (4.10), this shows (2.6), as desired.

Step 2. Proof of (2.7).

The starting point is once more the Green representation formula (4.1), from which we learn that

$$
\begin{array}{r}
\operatorname{var}\left[\int_{\varepsilon \mathbb{Z}^{d}} g(x) u_{\varepsilon}(x) d x\right]=\varepsilon^{4} \varepsilon^{2 d} \int_{\mathbb{Z}^{d}} \int_{\mathbb{Z}^{d}} \int_{\mathbb{Z}^{d}} \int_{\mathbb{Z}^{d}} g_{\varepsilon}(x) g_{\varepsilon}\left(x^{\prime}\right) f_{\varepsilon}(y) f_{\varepsilon}\left(y^{\prime}\right) \\
\operatorname{cov}\left[G_{\varepsilon^{2}}(x, y) ; G_{\varepsilon^{2}}\left(x^{\prime}, y^{\prime}\right)\right] d y d y^{\prime} d x d x^{\prime}
\end{array}
$$

where we have set $f_{\varepsilon}(x):=f(\varepsilon x)$ and $g_{\varepsilon}(x):=g(\varepsilon x)$ for all $x \in \mathbb{Z}^{d}$. We then appeal to the covariance estimate of Lemma 2.5 and to the estimate of the susceptibility of the Green's function in Lemma 2.7 to turn this into

$$
\begin{aligned}
& \operatorname{var}\left[\int_{\varepsilon \mathbb{Z}^{d}} g(x) u_{\varepsilon}(x) d x\right] \\
& \lesssim \varepsilon^{4} \varepsilon^{2 d} \int_{\mathbb{Z}^{d}} \int_{\mathbb{Z}^{d}} \int_{\mathbb{Z}^{d}} \int_{\mathbb{Z}^{d}}\left|g_{\varepsilon}(x) g_{\varepsilon}\left(x^{\prime}\right) f_{\varepsilon}(y) f_{\varepsilon}\left(y^{\prime}\right)\right| \\
& \quad \times\left\langle\int_{\mathbb{Z}^{d}}\left|\nabla_{z} G_{\varepsilon^{2}}(x, z)\right|\left|\nabla_{z} G_{\varepsilon^{2}}(z, y)\right|\left|\nabla_{z} G_{\varepsilon^{2}}\left(x^{\prime}, z\right)\right|\left|\nabla_{z} G_{\varepsilon^{2}}\left(z, y^{\prime}\right)\right| d z\right\rangle d y d y^{\prime} d x d x^{\prime} .
\end{aligned}
$$

We then let $2<p \leq 4$ be the minimum of 4 and of the Meyers exponent of Lemma 2.8. Hölder's inequality in probability with exponents $(2 p /(p-2), p, 2 p /(p-2), p)$ yields

$$
\begin{array}{r}
\operatorname{var}\left[\int_{\varepsilon \mathbb{Z}^{d}} g(x) u_{\varepsilon}(x) d x\right] \\
\lesssim \varepsilon^{4} \varepsilon^{2 d} \int_{\mathbb{Z}^{d}} \int_{\mathbb{Z}^{d}} \int_{\mathbb{Z}^{d}}\left[\int_{\mathbb{Z}^{d}} \int_{\mathbb{Z}^{d}}\left|g_{\varepsilon}(x) g_{\varepsilon}\left(x^{\prime}\right)\right|\left\langle\left|\nabla_{z} G_{\varepsilon^{2}}(x, z)\right|^{2 p /(p-2)}\right\rangle^{(p-2) /(2 p)}\right. \\
\left.\times\left\langle\left|\nabla_{z} G_{\varepsilon^{2}}\left(x^{\prime}, z\right)\right|^{2 p /(p-2)}\right\rangle^{(p-2) /(2 p)} d x d x^{\prime}\right] \\
\quad \times\left|f_{\varepsilon}(y) f_{\varepsilon}\left(y^{\prime}\right)\right|\left\langle\left|\nabla_{z} G_{\varepsilon^{2}}(z, y)\right|^{p}\right\rangle^{1 / p}\left\langle\left|\nabla_{z} G_{\varepsilon^{2}}\left(z, y^{\prime}\right)\right|^{p}\right\rangle^{1 / p} d z d y d y^{\prime} .
\end{array}
$$

We first deal with the term into brackets. By stationarity and Cauchy-Schwarz' inequality, for all $z \in \mathbb{Z}^{d}$,

$$
\begin{aligned}
& \int_{\mathbb{Z}^{d}} \int_{\mathbb{Z}^{d}}\left|g_{\varepsilon}(x) g_{\varepsilon}\left(x^{\prime}\right)\right|\left\langle\left|\nabla_{z} G_{\varepsilon^{2}}(x, z)\right|^{2 p /(p-2)}\right\rangle^{(p-2) /(2 p)}\left\langle\left|\nabla_{z} G_{\varepsilon^{2}}\left(x^{\prime}, z\right)\right|^{2 p /(p-2)}\right\rangle^{(p-2) /(2 p)} d x d x^{\prime} \\
& \quad \leq\|g\|_{\infty}^{2} \int_{\mathbb{Z}^{d}} \int_{\mathbb{Z}^{d}}\left\langle\left|\nabla_{z} G_{\varepsilon^{2}}(x, z)\right|^{2 p /(p-2)}\right\rangle^{(p-2) /(2 p)}\left\langle\left|\nabla_{z} G_{\varepsilon^{2}}\left(x^{\prime}, z\right)\right|^{2 p /(p-2)}\right\rangle^{(p-2) /(2 p)} d x d x^{\prime} \\
& \quad=\|g\|_{\infty}^{2}\left(\int_{\mathbb{Z}^{d}}\left\langle\left|\nabla_{x} G_{\varepsilon^{2}}(0, x)\right|^{2 p /(p-2)}\right\rangle^{(p-2) /(2 p)} d x\right)^{2} .
\end{aligned}
$$


Proceeding as for the proof of (4.9), we then deduce

$$
\begin{aligned}
& \int_{\mathbb{Z}^{d}} \int_{\mathbb{Z}^{d}}\left|g_{\varepsilon}(x) g_{\varepsilon}\left(x^{\prime}\right)\right|\left\langle\left|\nabla_{z} G_{\varepsilon^{2}}(x, z)\right|^{2 p /(p-2)}\right\rangle^{(p-2) /(2 p)} \\
& \times\left\langle\left|\nabla_{z} G_{\varepsilon^{2}}\left(x^{\prime}, z\right)\right|^{2 p /(p-2)}\right\rangle^{(p-2) /(2 p)} d x d x^{\prime} \\
& \lesssim\|g\|_{\infty}^{2}\left\{\begin{array}{lll}
d=2 & : & \varepsilon^{-2-(1-\gamma) \max \{0,4-p\}}|\ln \varepsilon|^{\max \{0,4-p\}}, \\
d>2 & : & \varepsilon^{-2-(1-\gamma) \max \{0,4-p\}} .
\end{array}\right.
\end{aligned}
$$

Let us quickly give the argument for $d>2$. Without loss of generality we assume that $p \leq 4$. Bounding $g_{\varepsilon}$ by its supremum, we are left with

$$
\begin{aligned}
\int_{\mathbb{Z}^{d}} \int_{\mathbb{Z}^{d}}\left|g_{\varepsilon}(x) g_{\varepsilon}\left(x^{\prime}\right)\right| & \left\langle\left|\nabla_{z} G_{\varepsilon^{2}}(x, z)\right|^{2 p /(p-2)}\right\rangle^{(p-2) /(2 p)} \\
& \times\left\langle\left|\nabla_{z} G_{\varepsilon^{2}}\left(x^{\prime}, z\right)\right|^{2 p /(p-2)}\right\rangle^{(p-2) /(2 p)} d x d x^{\prime} \\
& \lesssim\|g\|_{\infty}^{2}\left(\int_{\mathbb{Z}^{d}}\left\langle\left|\nabla_{z} G_{\varepsilon^{2}}(0, z)\right|^{2 p /(p-2)}\right\rangle^{(p-2) /(2 p)} d z\right)^{2}
\end{aligned}
$$

A dyadic decomposition of $\mathbb{Z}^{d}$ then yields

$$
\begin{gathered}
\int_{\mathbb{Z}^{d}}\left\langle\left|\nabla_{z} G_{\varepsilon^{2}}(0, z)\right|^{2 p /(p-2)}\right\rangle^{(p-2) /(2 p)} d z \leq \int_{|z| \leq R}\left\langle\left|\nabla_{z} G_{\varepsilon^{2}}(0, z)\right|^{2 p /(p-2)}\right\rangle^{(p-2) /(2 p)} d z \\
+\sum_{i=1}^{\infty} \int_{2^{i}<|z| \leq 2^{i+1} R}\left\langle\left|\nabla_{z} G_{\varepsilon^{2}}(0, z)\right|^{2 p /(p-2)}\right\rangle^{(p-2) /(2 p)} d z .
\end{gathered}
$$

The first term of the r. h. s. is of order 1 by Lemma 2.9. Proceeding as for (4.9), we first use Lemma 2.9, so that the integrals on dyadic annuli are estimated by

$$
\begin{aligned}
& \int_{2^{i}<|z| \leq 2^{i+1} R}\left\langle\left|\nabla_{z} G_{\varepsilon^{2}}(0, z)\right|^{2 p /(p-2)}\right\rangle^{(p-2) /(2 p)} d z \\
& \left.\left.\quad \lesssim \int_{2^{i}<|z| \leq 2^{i+1} R}\left(\frac{1}{1+|z|^{d+\gamma-2}} \exp (-c \varepsilon|z|)\right)^{(4-p) / 2}\langle| \nabla_{z} G_{\varepsilon^{2}} 0, z\right)\left.\right|^{p}\right\rangle^{(p-2) /(2 p)} d z .
\end{aligned}
$$

Combined with Hölder's inequality with exponents $(2 p /(p+2), 2 p /(p-2))$ and the estimate (2.26) of Lemma 2.8, this turns into

$$
\begin{aligned}
& \int_{2^{i} R<|z| \leq 2^{i+1} R}\left\langle\left|\nabla_{z} G_{\varepsilon^{2}}(0, z)\right|^{2 p /(p-2)}\right\rangle^{(p-2) /(2 p)} d x \\
& \quad \lesssim\left(2^{i}\right)^{(2-d-\gamma)(4-p) / 2+d(p+2) /(2 p)} \exp \left(-c \varepsilon 2^{i}\right)\left\langle\int_{2^{i} R \leq|z|<2^{i+1} R}\left|\nabla_{z} G_{\varepsilon^{2}}(0, z)\right|^{p} d x\right\rangle^{(p-2) /(2 p)} \\
& \quad \stackrel{(2.26)}{\lesssim}\left(2^{i}\right)^{(2-d-\gamma)(4-p) / 2+d(p+2) /(2 p)} \exp \left(-c \varepsilon 2^{i}\right)\left(2^{i}\right)^{(d+(1-d) p)(p-2) /(2 p)} \\
& \quad=\left(2^{i}\right)^{\frac{1}{2}(2+(1-\gamma)(4-p))} \exp \left(-c \varepsilon 2^{i}\right) .
\end{aligned}
$$

Summing over $i$ yields (4.16) for $d>2$, whereas the adaptation for $d=2$ is treated as for (4.9). 
We now treat the remaining term in (4.15): by symmetry of $y$ and $y^{\prime}$, and by stationarity

$$
\begin{aligned}
& \int_{\mathbb{Z}^{d}} \int_{\mathbb{Z}^{d}}\left|f_{\varepsilon}(y) f_{\varepsilon}\left(y^{\prime}\right)\right| \int_{\mathbb{Z}^{d}}\left\langle\left|\nabla_{z} G_{\varepsilon^{2}}(z, y)\right|^{p}\right\rangle^{1 / p}\left\langle\left|\nabla_{z} G_{\varepsilon^{2}}\left(z, y^{\prime}\right)\right|^{p}\right\rangle^{1 / p} d z d y d y^{\prime} \\
& \quad \leq \int_{\mathbb{Z}^{d}}\left|f_{\varepsilon}(y)\right|^{2} d y\left[\int_{\mathbb{Z}^{d}} \int_{\mathbb{Z}^{d}}\left\langle\left|\nabla_{z} G_{\varepsilon^{2}}(z, 0)\right|^{p}\right\rangle^{1 / p}\left\langle\left|\nabla_{z} G_{\varepsilon^{2}}\left(z, y^{\prime}\right)\right|^{p}\right\rangle^{1 / p} d z d y^{\prime}\right] .
\end{aligned}
$$

For the term into brackets, we use the estimate (4.13), so that

$$
\begin{aligned}
\int_{\mathbb{Z}^{d}} \int_{\mathbb{Z}^{d}}\left|f_{\varepsilon}(y) f_{\varepsilon}\left(y^{\prime}\right)\right| \int_{\mathbb{Z}^{d}} & \left\langle\left|\nabla_{z} G_{\varepsilon^{2}}(z, y)\right|^{p}\right\rangle^{1 / p} \\
\times & \left\langle\left|\nabla_{z} G_{\varepsilon^{2}}\left(z, y^{\prime}\right)\right|^{p}\right\rangle^{1 / p} d z d y d y^{\prime} \lesssim \varepsilon^{-d-2}\|f\|_{2}^{2} .
\end{aligned}
$$

The weak estimate (2.7) now follows from the combination of (4.15), (4.16), and (4.17).

4.2. Proof of Lemma 2.9. The diagonal estimate (that is for $|x-y| \lesssim 1$ ) follows from [7, Corollary 2.3]. The rest of the proof is similar to the proof of Lemma 2.4. It relies on a Harnack inequality for positive solutions of discrete elliptic equations on $\mathbb{Z}^{d}$ combined with the pointwise decay of the Green's function itself. W. l. o. g. we assume $\eta \gg 1$.

A discrete version of Cacciopoli's estimate (see for instance [7, Proof of Lemma 2.8]) yields for all $|z-y| \gg 1$ and all $k \in \mathbb{R}$ :

$$
\int_{B_{R}(z)}\left|\nabla_{x} G_{\eta}(x, y)\right|^{2} d x \lesssim \int_{B_{2 R}(z)}\left(G_{\eta}(x, y)-k\right)^{2} d x
$$

and therefore

$$
\left|\nabla_{x} G_{\eta}(z, y)\right| \lesssim \underset{x \in B_{2 R}(z)}{\operatorname{osc}} G_{\eta}(x, y)
$$

by choosing $k=\inf _{x \in B_{2 R}(z)} G_{\eta}(x, y)$ and using the discrete $L^{2}-L^{\infty}$ estimate. We then appeal to a discrete version of the Hölder regularity of [4, Theorem 8.22], which follows for instance from [13]: There exists $0<\gamma<1$ depending only on $\alpha, \beta$ and $d$ such that for all $r>d$,

$$
\operatorname{osc}_{x \in B_{2 R}(z)} G_{\eta}(x, y) \lesssim\left(|z-y|^{-\gamma}+\eta|z-y|^{d / r}\right) \sup _{x \in B_{|z-y| / 2}(z)} G_{\eta}(x, y),
$$

where the multiplicative constant depends on $r$ next to $\alpha, \beta$, and $d$. For $|z-y| \leq \eta^{-1}$, (4.19) turns into

$\underset{x \in B_{2 R}^{\mathrm{OSC}}(z)}{\operatorname{Sin}} G_{\eta}(z, y) \lesssim|z-y|^{2-d-\gamma} \begin{cases}d=2 & : \ln ((1+|z-y|) \sqrt{\eta}) \exp (-c|z-y| \sqrt{\eta}), \\ d>2 & : \exp (-c|z-y| \sqrt{\eta}),\end{cases}$

choosing $r=\frac{d}{1-\gamma}$ and using in addition the pointwise estimates of [5, Lemma 2]. For $|z-y|>\eta$, choosing $r=d+1$ and using that

$$
\exp (-c t \sqrt{\eta}) \lesssim t^{2} \eta \exp \left(-\frac{c}{2} t \sqrt{\eta}\right),
$$

(4.19) also turns into (4.20) (possibly with a smaller $c>0$ ). The claim of the lemma follows from the combination of (4.18) and (4.20), and the bound $\left|\nabla G_{\eta}\right| \lesssim 1$ from [7, Corollary 2.3]. 


\section{Appendix A. Covariance estimate in the continuum Case}

\section{A.1. Statement.}

Lemma A.1 (covariance estimate). Let $A$ be random with values in $\mathcal{A}_{\alpha \beta}$ with the following statistical property: The random field $A$ has correlation length unity in the sense that for any two sets $U, V \subset \mathbb{R}^{d}$ with $\operatorname{dist}(U, V) \geq 1 / 3$, the restrictions $A_{\mid U}$ and $A_{\mid V}$ are independent.

Let $X$ and $Y$ be functions on $\mathcal{A}_{\alpha \beta}$ - which we consider as a random variable - with the following regularity property: $X$ and $Y$ can be uniformly approximated by functions $\tilde{X}$ and $\tilde{Y}$ on $\mathcal{A}_{\alpha \beta}$ that depend on $A$ only through the restriction $A_{\mid \tilde{U}}$ for some compact set $\tilde{U} \subset \mathbb{R}^{d}$.

Then we have the following covariance estimate

$$
\operatorname{cov}[X ; Y] \leq \int_{\mathbb{R}^{d}}\left\langle\begin{array}{cc}
\operatorname{Osc}^{2} & \left.X\right|_{Q(z)}
\end{array}\right\rangle^{1 / 2}\left\langle\begin{array}{cc}
\operatorname{Osc}^{2} & \left.Y\right|_{Q(z)}
\end{array}\right\rangle^{1 / 2} d z,
$$

where for $Z=X, Y$, osc $Z$ denotes the oscillation of $Z$ with respect to $A$ restricted onto $\left.A\right|_{Q_{(z)}}$

the cube $Q(z)$ of lateral size unity and center at $z \in \mathbb{R}^{d}$. Note that for some set $U \subset \mathbb{R}^{d}$, osc $Z$ itself is a random variable: $\left.A\right|_{U}$

$$
\begin{aligned}
\left(\begin{array}{c}
\operatorname{osc} Z \\
\left.A\right|_{U}
\end{array}\right)(A)= & \left(\sup _{\left.A\right|_{U}} Z\right)(A)-\left(\inf _{\left.A\right|_{U}} Z\right)(A) \\
= & \sup \left\{Z(\tilde{A})\left|\tilde{A} \in \mathcal{A}_{\alpha \beta}, \tilde{A}\right|_{\mathbb{R}^{d}-U}=\left.A\right|_{\mathbb{R}^{d}-U}\right\} \\
& -\inf \left\{Z(\tilde{A})\left|\tilde{A} \in \mathcal{A}_{\alpha \beta}, \tilde{A}\right|_{\mathbb{R}^{d}-U}=\left.A\right|_{\mathbb{R}^{d}-U}\right\} .
\end{aligned}
$$

A.2. Proof. We decompose the proof of the covariance estimate into four steps.

Step 1. Preliminaries.

We shall establish the following somewhat stronger version of (A.1): For any lateral cube size $h>0$ we have

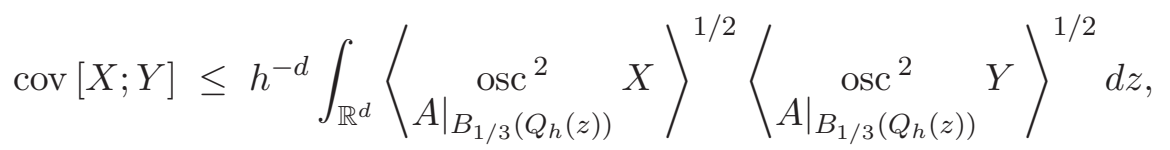

where $B_{1 / 3}(U)$ denotes the $1 / 3$-neighborhood of a set $U \subset \mathbb{R}^{d}$, that is $B_{1 / 3}(U)=\{x \in$ $\left.\mathbb{R}^{d} \mid \operatorname{dist}(x, U) \leq 1 / 3\right\}$. Note that by averaging over translations in $Q_{h}(0)$, (A.3) can be derived from its discrete version

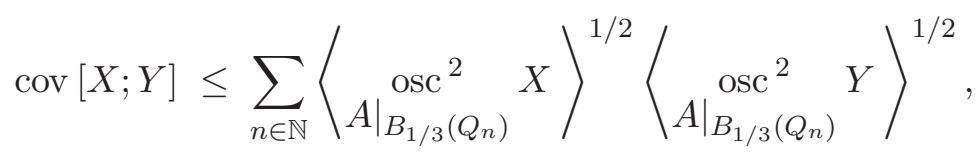

where $\left\{Q_{n}\right\}_{n \in \mathbb{N}}$ is a partitioning of $\mathbb{R}^{d}$ into cubes of lateral size $h$ (in some arbitrary enumeration). In fact, we will prove (A.4) for any countable covering $\left\{Q_{n}\right\}_{n \in \mathbb{N}}$ of $\mathbb{R}^{d}$ by open sets.

Step 2. Reformulation of the assumption on the statistics of $A$. 
To ease notation, we introduce the following language and abbreviations for a random variable $Z$ (i. e. a function of $A \in \mathcal{A}_{\alpha \beta}$ ) and a set $U \subset \mathbb{R}^{d}$ :

- we say $Z$ depends only on $U$ if $Z$ depends on $A$ only via $A_{\mid U}$,

- we say $Z$ does not depend on $U$ if $Z$ depends only on $\mathbb{R}^{d}-U$,

- $\sup _{U} Z, \inf _{U} Z$, and $\underset{U}{\operatorname{osc}} Z$ denote the the supremum, the infimum, and the oscillation, respectively, of $Z$ with respect to $A_{\mid U}$ in the sense of (A.2) - note that they do not depend on $U$,

- $\langle Z \mid U\rangle$ denotes the expectation of $Z$ conditioned on the values of $A_{\mid U}$ - note that it depends only on $U$.

With this notation, we can reformulate the assumption on the statistics of $A$ as follows: For any two sets $V, W \subset \mathbb{R}^{d}$ with $\operatorname{dist}(V, W) \geq 1 / 3$ and any random variable $Z$ we have the implication

$\mathrm{Z}$ depends only on $W$

$$
\Longrightarrow \forall U \subset \mathbb{R}^{d}: \quad\langle Z \mid U\rangle \text { does not depend on } V .
$$

We note that this implies for any two sets $U, V \subset \mathbb{R}^{d}$ :

$$
\underset{V}{\operatorname{osc}}\langle Z \mid U\rangle \leq \underset{B_{1}(V)}{\operatorname{osc}} Z|U\rangle
$$

- it is this inequality that is at the heart of (A.4). Indeed, by definition of the oscillation and by replacing $Z$ with $-Z$, it suffices to show

$$
\sup _{V}\langle Z \mid U\rangle \leq\left\langle\sup _{B_{1}(V)} Z \mid U\right\rangle
$$

Inequality (A.7) can derived from (A.5) as follows: Clearly, the random variable $\sup _{B_{1 / 3}(V)} X$ depends only on the set $W:=\mathbb{R}^{d}-B_{1}(V)$ so that by (A.5) the random variable $\left\langle\sup _{B_{1 / 3}(V)} Z \mid U\right\rangle$ does not depend on $V$. Therefore (A.7) follows from the obvious inequality $\langle Z \mid U\rangle \leq$ $\left\langle\sup _{B_{1 / 3}(V)} Z \mid U\right\rangle$.

Step 3. Lu-Yau martingale method.

Based on the (arbitrary) enumeration of the countable covering $\left\{Q_{n}\right\}_{n \in \mathbb{N}}$ of $\mathbb{R}^{d}$ by open sets, we introduce the sequences $\left\{X_{n}\right\}_{n \in \mathbb{N}}$ and $\left\{Y_{n}\right\}_{n \in \mathbb{N}}$ of random variables

$$
X_{n}:=\left\langle X \mid Q_{1} \cup \cdots \cup Q_{n}\right\rangle, \quad Y_{n}:=\left\langle Y \mid Q_{1} \cup \cdots \cup Q_{n}\right\rangle,
$$

with the convention that $X_{0}:=\langle X\rangle$ and $Y_{0}:=\langle Y\rangle$. By our regularity assumption on $X$ and $Y$, we may assume that $X$ and $Y$ only depend on $A$ via $A_{\mid U_{0}}$ for some possibly large but compact set $U_{0}$. Since $U_{0}$ is covered by finitely many of the $\left\{Q_{n}\right\}_{n \in \mathbb{N}}$, there exists a possible large but finite $N \in \mathbb{N}$ such that $X_{N}=X$ and $Y_{N}=Y$. This yields for the covariance

$$
\operatorname{cov}[X, Y ;]=\langle X Y\rangle-\langle X\rangle\langle Y\rangle=\left\langle X_{N} Y_{N}\right\rangle-\left\langle X_{0} Y_{0}\right\rangle .
$$

Hence, in order to establish (A.4), it suffices to show for arbitrary $n \in \mathbb{N}$ :

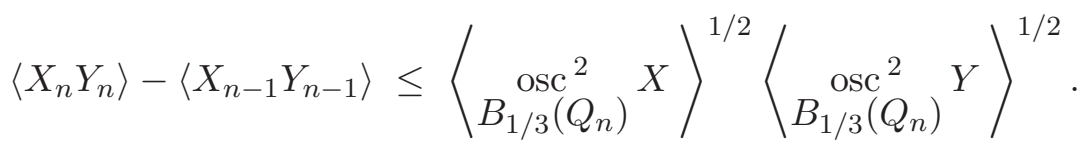

Step 4. Derivation of (A.9) from (A.6). 
We actually establish the more detailed estimate

$$
\begin{aligned}
& \left\langle X_{n} Y_{n} \mid Q_{1} \cup \cdots \cup Q_{n-1}\right\rangle-\left\langle X_{n-1} Y_{n-1} \mid Q_{1} \cup \cdots \cup Q_{n-1}\right\rangle \\
& \quad \leq\left\langle\underset{B_{1 / 3}\left(Q_{n}\right)}{\operatorname{osc}^{2}} X \mid Q_{1} \cup \cdots \cup Q_{n-1}\right\rangle^{1 / 2}\left\langle\underset{B_{1 / 3}\left(Q_{n}\right)}{\operatorname{osc}^{2}} Y \mid Q_{1} \cup \cdots \cup Q_{n-1}\right\rangle^{1 / 2}
\end{aligned}
$$

We note that by definition (A.8) of $\left\{X_{n}\right\}_{n \in \mathbb{N}}$ and $\left\{Y_{n}\right\}_{n \in \mathbb{N}}$ :

$$
\begin{aligned}
\left\langle X_{n-1} Y_{n-1} \mid Q_{1} \cup \cdots \cup Q_{n-1}\right\rangle & =X_{n-1} Y_{n-1} \\
& =\left\langle X_{n} \mid Q_{1} \cup \cdots \cup Q_{n-1}\right\rangle\left\langle Y_{n} \mid Q_{1} \cup \cdots \cup Q_{n-1}\right\rangle,
\end{aligned}
$$

so that the left hand side of (A.10) can be written as a covariance:

$$
\begin{aligned}
& \operatorname{cov}\left[X_{n} ; Y_{n} \mid Q_{1} \cup \cdots \cup Q_{n-1}\right] \\
& \leq\left\langle\underset{B_{1 / 3}\left(Q_{n}\right)}{\operatorname{osc}^{2}} X \mid Q_{1} \cup \cdots \cup Q_{n-1}\right\rangle^{1 / 2}\left\langle\underset{B_{1 / 3}\left(Q_{n}\right)}{\operatorname{osc}^{2}} Y \mid Q_{1} \cup \cdots \cup Q_{n-1}\right\rangle^{1 / 2} .
\end{aligned}
$$

By Jensen's inequality on its right hand side, the latter inequality follows from the following for $Z=X, Y$ :

$$
\left|Z_{n}-\left\langle Z_{n} \mid Q_{1} \cup \cdots \cup Q_{n-1}\right\rangle\right| \leq\left\langle\underset{B_{1 / 3}\left(Q_{n}\right)}{\operatorname{osc}} Z \mid Q_{1} \cup \cdots \cup Q_{n}\right\rangle .
$$

By replacing $Z$ by $-Z$, it is enough to show

$$
Z_{n}-\left\langle Z_{n} \mid Q_{1} \cup \cdots \cup Q_{n-1}\right\rangle \leq\left\langle\underset{B_{1 / 3}\left(Q_{n}\right)}{\operatorname{osc}} Z \mid Q_{1} \cup \cdots \cup Q_{n}\right\rangle .
$$

After these reformulations, it is easy to see that (A.11) follows from (A.6):

$$
\begin{aligned}
Z_{n} & -\left\langle Z_{n} \mid Q_{1} \cup \cdots \cup Q_{n-1}\right\rangle \\
& \leq \quad \sup _{Q_{n}} Z_{n}-\left\langle\inf _{Q_{n}} Z_{n} \mid Q_{1} \cup \cdots \cup Q_{n-1}\right\rangle \\
& =\sup _{Q_{n}} Z_{n}-\inf _{Q_{n}} Z_{n} \\
\stackrel{(A .8)}{=} & \operatorname{OSc}_{Q_{n}}\left\langle Z \mid Q_{1} \cup \cdots \cup Q_{n}\right\rangle \\
\stackrel{(A .6)}{\leq} & \left\langle\quad \operatorname{osc}_{B_{1 / 3}\left(Q_{n}\right)} Z \mid Q_{1} \cup \cdots \cup Q_{n}\right\rangle .
\end{aligned}
$$

\section{REFERENCES}

[1] G. Bal. Central limits and homogenization in random media. Multiscale Model. Simul., 7:677-702, 2008.

[2] J.G. Conlon and A. Naddaf. On homogenization of elliptic equations with random coefficients. Elec. Journal of Probability, 5:1-58, 2000. Paper no. 9.

[3] R. Figari, E. Orlandi, and G. Papanicolaou. Mean field and Gaussian approximation for partial differential equations with random coefficients. SIAM J. Appl. Math., 42:1069-1077, 1982.

[4] D. Gilbarg and N.S. Trudinger. Elliptic partial differential equations of second order. Classics in Mathematics. Springer-Verlag, Berlin, 2001. Reprint of the 1998 edition. 
[5] A. Gloria. Numerical approximation of effective coefficients in stochastic homogenization of discrete elliptic equations. M2AN Math. Model. Numer. Anal., 46(1):1-38, 2012.

[6] A. Gloria and F. Otto. Optimal quantitative estimates in stochastic homogenization of linear elliptic equations. In preparation.

[7] A. Gloria and F. Otto. An optimal variance estimate in stochastic homogenization of discrete elliptic equations. Ann. Probab., 39(3):779-856, 2011.

[8] A. Gloria and F. Otto. An optimal error estimate in stochastic homogenization of discrete elliptic equations. Ann. Appl. Probab., 22(1):1-28, 2012.

[9] M. Grüter and K.-O. Widman. The Green function for uniformly elliptic equations. Manuscripta Math., 37:303-342, 1982.

[10] K. Klenke. Wahrscheinlichkeitstheorie. Springer-Verlag, Heidelberg-Berlin, 2006. English version appeared as: Probability theory. A comprehensive course, Universitext, Springer-Verlag London, Ltd., London, 2008.

[11] W. Littman, G. Stampacchia, and H. F. Weinberger. Regular points for elliptic equations with discontinuous coefficients. Ann. Scuola Norm. Sup. Pisa (3), 17:43-77, 1963.

[12] N. Meyers. An $L^{p}$-estimate for the gradient of solutions of second order elliptic divergence equations. Ann. Scuola Norm. Sup. Pisa Cl. Sci. (3), 17(3):189-206, 1963.

[13] S.J.N. Mosconi. Discrete regularity for elliptic equations on graphs. CVGMT preprint, http://cvgmt.sns.it/cgi/get.cgi/papers/mos01, 2001.

[14] J. Nolen and G. Papanicolaou. Fine scale uncertainty in parameter estimation for elliptic equations. Inverse Problems, 25(11):115021, 22, 2009.

[15] G.C. Papanicolaou and S.R.S. Varadhan. Boundary value problems with rapidly oscillating random coefficients. In Random fields, Vol. I, II (Esztergom, 1979), volume 27 of Colloq. Math. Soc. János Bolyai, pages 835-873. North-Holland, Amsterdam, 1981.

[16] V. V. Yurinskil. Averaging of symmetric diffusion in random medium. Sibirskii Matematicheskii Zhurnal, 27(4):167-180, 1986.

(Antoine Gloria) Project-team Simpaf \& Laboratoire Paul Painlevé UMR 8524, inRIA Lille - Nord Europe \& Université Lille 1, Villeneuve D'AscQ, France

E-mail address: antoine.gloria@inria.fr 